\title{
Normal and anomalous solitons in the theory of dynamical Cooper pairing
}

\author{
Emil A. Yuzbashyan \\ Center for Materials Theory, Department of Physics and Astronomy, \\ Rutgers University, Piscataway, New Jersey 08854, USA
}

\begin{abstract}
We obtain multi-soliton solutions of the time-dependent Bogoliubov-de Gennes equations or, equivalently, Gorkov equations that describe the dynamics of a fermionic condensate in the dissipationless regime. There are two kinds of solitons - normal and anomalous. At large times, normal multi-solitons asymptote to unstable stationary states of the BCS Hamiltonian with zero order parameter (normal states), while the anomalous ones tend to eigenstates characterized by a nonzero anomalous average. Under certain circumstances, multi-soliton solutions break up into sums of single solitons. In the linear analysis near the stationary states, solitons correspond to unstable modes. Generally, they are nonlinear extensions of these modes, so that a stationary state with $k$ unstable modes gives rise to a $k$-soliton solution. We relate parameters of the multi-solitons to those of the asymptotic stationary state, which determines the conditions necessary for exciting solitons. We further argue that the dynamics in many physical situations is multi-soliton.
\end{abstract}




\section{Contents}

I. Introduction and summary of the results
A. Normal solitons
B. Anomalous solitons

II. Review of the basic setup and relevant previous results
A. Notations and basic equations
B. General properties of the dynamics

III. Linear analysis around stationary states

A. Frequencies of oscillations around stationary states

B. Examples of root diagrams of $\mathbf{L}^{2}(u)$

1. BCS ground state

2. Excited anomalous states

\section{Example 1}

Example 2

3. Fermi ground state

4. Excited normal state

IV. Normal solitons

A. General $k$-normal-soliton solution

B. Matching soliton constants to spin configuration at large negative time

C. Examples of 1 and 2-normal-solitons

\section{Anomalous solitons}

A. Single anomalous soliton as a special case of a 3-spin solution

B. 2-anomalous-soliton solutions

\section{Conclusion}

\section{Acknowledgements}

\section{References}




\section{INTRODUCTION AND SUMMARY OF THE RESULTS}

Recent years have witnessed renewed experimental and theoretical interest in far from equilibrium phenomena in strongly interacting many-body systems at low temperatures. Examples include non-stationary Kondo and other impurity models [1 9], quenched Luttinger liquids [10 13], electron spin dynamics induced by hyperfine interactions[14-22] etc. On the theory side, there is a considerable effort to develop new approaches to nonequilibrium many-body physics. This presents a significant challenge as conventional techniques are often inadequate for the description of these phenomena. In particular, there have been major advances in the theory of dynamical fermionic pairing in the collisionless regime 23 37]. This problem is long known to be accurately described by the time-dependent Bogoliubov-de Gennes equations, which in this case are a set of coupled nonlinear integro-differential equations [24, 25, 27, 30]. Nevertheless, it was not until recently that this nonlinear system was realized to be exactly solvable[29, 30, 33]. The exact solution proved to be a unique approach to the problem of dynamical pairing and has been extensively exploited to obtain analytical information about its key physical properties. For example, a nonequilibrium "phase diagram" of a homogeneous Bardeen-Cooper-Schriffer (BCS) superfluid with a number of novel phases, as well as their responses to existing experimental probes were predicted analytically [34, 35, 36, 37].

However, while much attention was focused on the asymptotic states of the condensate at large times, the transient dynamics has not been fully explored. Most importantly, nonlinear integrable systems are known to exhibit a remarkable class of multi-soliton solutions that play a central role in understanding and predicting their properties. Physical solutions can often be represented as a superposition of solitons making a quantitative analysis possible. For instance, one can show that the dynamics giving rise to nonequilibrium "phases" mentioned above is multi-soliton, see below. The existence and properties of solutions of this type are also of a general interests from the point of view of nonlinear physics due to the nonlocal nature of the BCS problem distinguishing it from familiar integrable systems, such as nonlinear Shrödinger, Korteweg-de Vries, sine-Gordon etc.

In this paper we construct multi-soliton solutions to the dynamical fermionic pairing problem, see Figs. 1 - 5. We establish a direct correspondence between solitons and the stationary states of the mean-field BCS Hamiltonian, such that each soliton solution asymptotes to an eigenstate at times $t \rightarrow \pm \infty$. This also identifies conditions necessary for exciting solitons. There are two distinct types of solitons - normal and anomalous. Normal solitons asymptote to stationary states that are simultaneous eigenstates of a Fermi gas and the mean-field BCS Hamiltonian and are 
characterized by a zero anomalous average. For anomalous solitons the asymptotic value of this average is finite, $\Delta(t \rightarrow \pm \infty) \neq 0$. As the separation between solitons is increased, the multisoliton solution breaks up into a simple sum of single solitons (see e.g. Figs. 2 and 3) - one of the defining properties of solitons.

In the rest of this section, we briefly formulate the problem and then summarize the main results. The collisionless dynamics of a fermionic superfluid can be described by the Bogoliubov-de Gennes equations [27, 30]

$$
i \frac{d}{d t}\left(\begin{array}{c}
U_{m} \\
V_{m}
\end{array}\right)=\left(\begin{array}{cc}
\epsilon_{m} & \Delta(t) \\
\Delta^{*}(t) & -\epsilon_{m}
\end{array}\right)\left(\begin{array}{c}
U_{m} \\
V_{m}
\end{array}\right)
$$

where $\Delta(t)=g \sum_{m} U_{m} V_{m}^{*}$ is the anomalous average, $\epsilon_{m}$ are the single fermion energies relative to the Fermi level $\epsilon_{F}$, and $g$ is the coupling constant. These nonlinear equations are known to be integrable for any number of Bogoliubov amplitudes $\left(U_{m}, V_{m}\right)[29$, 30, 33]. In the continuum limit the summation in the expression for $\Delta(t)$ is replaced by integration and Eqs. (1) become integrable nonlinear integro-differential equations. Each solution of Eq. (1) yields a many-body wave function

$$
|\Psi(t)\rangle=\prod_{m}\left[U_{m}^{*}(t)+V_{m}^{*}(t) \hat{c}_{m \uparrow}^{\dagger} \hat{c}_{m \downarrow}^{\dagger}\right]|0\rangle,
$$

where the product is taken only over unblocked levels - levels that are either unoccupied or doubly occupied.

As we demonstrate in subsequent sections, solitons tend to unstable stationary states of the mean-field BCS Hamiltonian at $t \rightarrow \pm \infty$. The mean-field Hamiltonian has two types of eigenstates - normal and anomalous. Anomalous stationary states have a nonzero constant value $\Delta_{a}$ of the anomalous average and are solutions of Eq. (11) of the form [38, 39] $\left(U_{m}, V_{m}\right)=\left(U_{m}^{0}, V_{m}^{0}\right) e^{-i E_{m} t}$, where $\left(U_{m}^{0}, V_{m}^{0}\right)$ and $E_{m}$ are the eigenvectors and eigenvalues of the $2 \times 2$ matrix on the right hand side of Eq. (11). There are two states $E_{m}= \pm \sqrt{\epsilon_{m}^{2}+\Delta^{2}}$ for each $\epsilon_{m}$. The BCS ground state has $E_{m}<0$ for all $m$. A state where $E_{r}>0$ while $E_{m \neq r}<0$ describes a single excited pair[38, 40] and has energy $2 \sqrt{\epsilon_{r}^{2}+\Delta^{2}}$ above the ground state. We note also that an excited pair introduces a discontinuity in the average fermion occupation number $n\left(\epsilon_{m}\right)=1-\epsilon_{m} / E_{m}$, since $E_{m}$ changes sign at $m=r$. Normal eigenstates have $\Delta=0$ and amplitudes $\left(U_{m}, V_{m}\right)$ equal to either $\left(0, e^{i \epsilon_{m} t}\right)$ or $\left(e^{-i \epsilon_{m} t}, 0\right)$. Both types of eigenstates are exact stationary states of the mean-field BCS Hamiltonian

$$
\hat{H}=\sum_{j ; \sigma=\downarrow, \uparrow} \epsilon_{j} \hat{c}_{j \sigma}^{\dagger} \hat{c}_{j \sigma}-\sum_{j, k}\left(\Delta \hat{c}_{j \uparrow}^{\dagger} \hat{c}_{j \downarrow}^{\dagger}+\text { h.c. }\right),
$$

where $\Delta=g \sum_{k}\left\langle\hat{c}_{k \downarrow} \hat{c}_{k \uparrow}\right\rangle$, and $\hat{c}_{j \sigma}^{\dagger}$ and $\hat{c}_{j \sigma}$ are the creation and annihilation operators for the two fermion species. Normal states are also eigenstates of the Fermi gas - the first term in Eq. (3). For 
example, the Fermi ground state is a normal eigenstate with $U_{m}=0$ for $\epsilon_{m}<0$ and $V_{m}=0$ for $\epsilon_{m}>0$, i.e. all single particle states below the Fermi level occupied and states above it empty.

A linear analysis of Eq. (11) around stationary states shows that some of them are unstable 27, 41]. These states give rise to solitons, as is typical in integrable nonlinear dynamics. For example, a simple pendulum displays a soliton solution when started in its unstable equilibrium with zero velocity. In the phase space the soliton is the separatrix connecting the unstable equilibrium to itself. The same is true for example for the single soliton solution of the Korteweg-de Vries equation[42]. Similarly in the present case the unstable modes start to grow exponentially and become solitons due to nonlinear effects. In a certain sense, solitons can be viewed as nonlinear extensions of the unstable modes.

Now let us summarize soliton solutions of the Bogoliubov-de Gennes equations (1). Detailed derivation and discussion of these as well as some other solutions can be found in subsequent sections. Here we present only the results for the amplitude of the order parameter $|\Delta(t)|$.

\section{A. Normal solitons}

First, we present normal multi-solitons derived in Sec. IV] The 1-soliton solution of a normal type has been previously obtained in Ref 27. The amplitude of the order parameter has the following form:

$$
|\Delta(t)|=\frac{2 \gamma}{\cosh (2 \gamma t+\alpha)}
$$

where $\alpha$ is a real parameter. At $t \rightarrow \pm \infty$ the corresponding wave function (2) asymptotes to the Fermi ground state. The latter is unstable in the presence of the pairing interaction. Indeed, a linear analysis of equations of motion (11) around the Fermi ground state shows a single unstable normal mode, which grows as $|\Delta(t)| \propto e^{2 \gamma t}[27$, 41] as can be seen from Eq. (44) in the $t \rightarrow-\infty$ limit. The plot of Eq. (4) is a single peak centered at $t=-\alpha / 2 \gamma$, see Fig. 11. Its height (the amplitude of the soliton) and width are controlled by the parameter $2 \gamma$. In the present case $2 \gamma=\Delta_{0}$, where $\Delta_{0}$ is the ground state BCS gap. This parameter can be interpreted as an imaginary "frequency" of the unstable normal mode, while Eq. (4) as an extension of this mode to the nonlinear regime. Below we will see that the number of unstable modes and consequently the number of solitons corresponding to a given stationary state is related to the number of discontinuities in the average fermion occupation number $n(\epsilon)$ in this state. Specifically, $2 k-1$ discontinuities (jumps) in $n(\epsilon)$ lead to up to $k$ coupled normal solitons. The Fermi ground state has a single discontinuity at the 


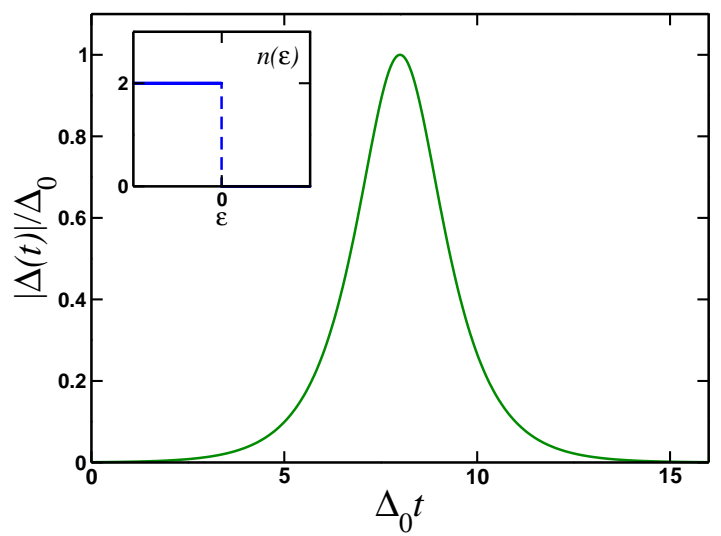

FIG. 1: (color online) $k=1$ normal-soliton solution of the Bogoliubov-de Gennes equations (11) 27], where $\Delta_{0}$ is the ground state BCS gap. At $t \rightarrow \pm \infty$ the solution asymptotes to the Fermi ground state. The inset shows the average fermion occupation number $n(\epsilon)$ in this state. A single discontinuity $(2 k-1=1)$ in $n(\epsilon)$ at the Fermi energy gives rise to a single soliton, see the text.

Fermi level, giving rise to the 1-soliton solution.

The 2-normal-solitons have considerably richer structure. Let us give two examples. Both are described by

$$
|\Delta(t)|=A\left|\frac{h(t)}{h(t) \ddot{h}(t)-\dot{h}^{2}(t)}\right|,
$$

with different choices for the amplitude $A$ and function $h(t)$. One choice is $A=4\left|\gamma_{2}^{2}-\gamma_{1}^{2}\right|$ and

$$
h(t)=e^{i \phi_{1}} \frac{\cosh \left(2 \gamma_{1} t+\alpha_{1}\right)}{2 \gamma_{1}}+e^{i \phi_{2}} \frac{\cosh \left(2 \gamma_{2} t+\alpha_{2}\right)}{2 \gamma_{2}} .
$$

Examples of $|\Delta(t)|$ for this case are plotted in Fig. 22a. The second option is $A=16 \mu \sqrt{\mu^{2}+\gamma^{2}}$ and

$$
h(t)=e^{-2 i \mu t+i \phi_{1}} \frac{\cosh \left(2 \gamma t+\alpha_{1}-i \beta\right)}{2 \gamma}+e^{2 i \mu t+i \phi_{2}} \frac{\cosh \left(2 \gamma t+\alpha_{2}+i \beta\right)}{2 \gamma},
$$

where $\beta$ is the phase of $\mu+i \gamma$, i.e. $\mu+i \gamma=\sqrt{\mu^{2}+\gamma^{2}} e^{i \beta}$. Fig. $2 \mathrm{~b}$ shows graphs of $|\Delta(t)|$ obtained using Eq. (7). In both cases the wave function asymptotes to a normal eigenstate at $t \rightarrow \pm \infty$. This eigenstate is obtained from the Fermi ground state by moving all fermions in the energy interval $-a<\epsilon_{m}<0$ below the Fermi level to the symmetric interval $0<\epsilon_{m}<a$ above it. Eqs. (66) and (77) correspond to different values of $a$. Note that this normal eigenstate has $2 k-1=3$ discontinuities at $\epsilon=-a$, 0, and $a$ in $n(\epsilon)$ (see the insets in Fig. 2) thus leading to $k=2$ solitons. A linear analysis around this state yields two unstable modes that exponentially grow with rates $2 \gamma_{1,2}$ in the first case and $2 \gamma \pm 2 i \mu$ in the second. Which of the two cases is realized depends on the ratio 

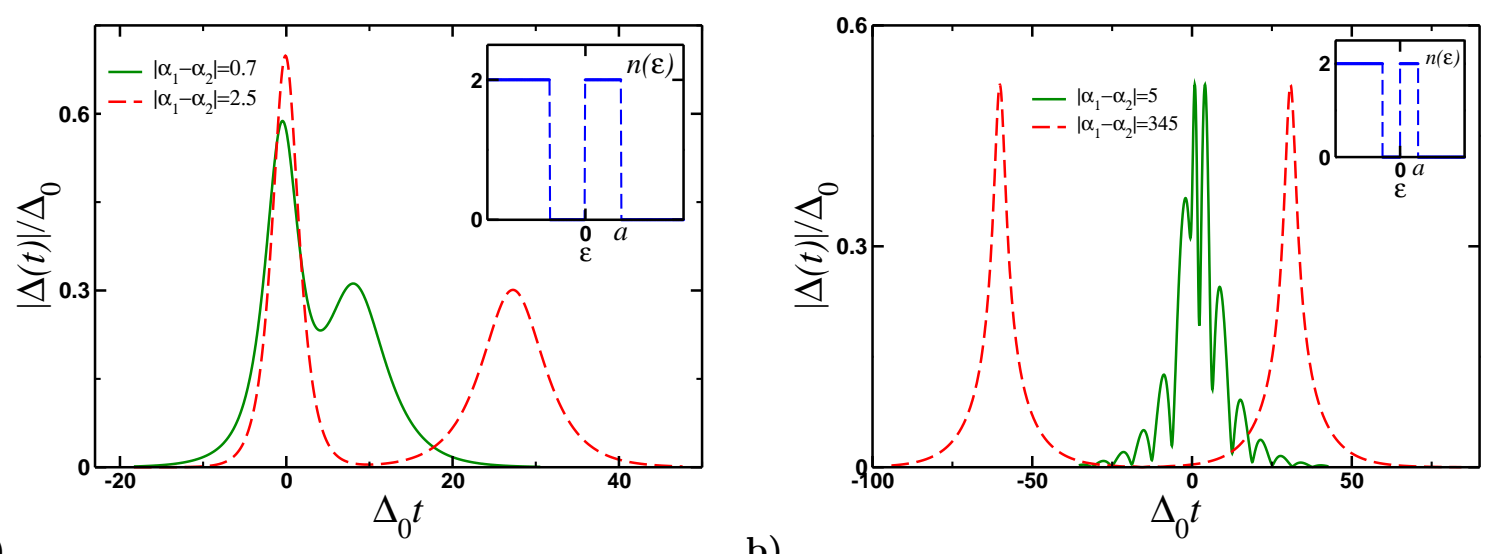

a)

b)

FIG. 2: (color online) $k=2$ normal soliton solutions to the Bogoliubov-de Gennes equations (11). $\Delta_{0}$ is the ground state BCS gap. At $t \rightarrow \pm \infty$ the 2 -solitons tend to a normal state characterized by $2 k-1=3$ jumps at $\epsilon=0$ and $\pm a$ in the average fermion occupation number $n(\epsilon)$ (insets). a) is obtained from Eq. (6), where $\gamma_{1,2}$ are related to $a$ and $\Delta_{0}$ by Eq. (55), and $4.25 a=\Delta_{0}$. b) corresponds to Eq. (7) with $4 \mu=\Delta_{0}$, $4 \gamma=\sqrt{16 a^{2}-\Delta_{0}^{2}}$ (see the text below Eq. (55)), and $a=3.75 \Delta_{0} . \phi_{1,2}=0$ in both cases. For large separation, $\left|\alpha_{1}-\alpha_{2}\right| \gg 1$, the 2-soliton solutions split into two single solitons (dashed lines), see Eqs. (44), (12), (90), and Fig. 1. For $\left|\alpha_{1}-\alpha_{2}\right| \rightarrow 0$ the two solitons merge into a single peak. In b) the amplitude of this peak is modulated with a frequency $\omega \simeq 4 \mu=\Delta_{0}$ as the two terms in Eq. (7) "rotate" with respect to each other.

$a / \Delta_{0}$. The values of $\gamma_{1,2}$ or $\gamma \pm i \mu$ are also fixed by $a$ and $\Delta_{0}$, while $\alpha_{1,2}$ and $\phi_{1,2}$ are arbitrary real parameters. They control the separation between solitons and their relative phase in the 2-soliton solution, respectively. For sufficiently large $\left|\alpha_{1}-\alpha_{2}\right|$ Eq. (5) always breaks up into a sum of two single solitons of the form (4). The graph of $|\Delta(t)|$ in this case displays two well separated peaks, each representing a single soliton, see Fig. 2 .

The general $k$-soliton solution of the normal type has the form (see Sec. IVA)

$$
|\Delta(t)|=2^{2 k-1}\left|\frac{D_{k-1}}{D_{k}}\right|
$$

where $D_{r}$ is the following determinant:

$$
D_{r}=\left|\begin{array}{lll}
f & \ldots & f^{(r-1)} \\
\vdots & & \vdots \\
f^{(r-1)} & \ldots & f^{2(r-1)}
\end{array}\right|
$$

$f^{(m)}$ is the $m$ th derivative of the function $f(t)$ with respect to $t$, and

$$
f(t)=\sum_{j=1}^{2 k} \frac{A\left(c_{j}\right) e^{-2 i c_{j} t}}{\prod_{m \neq j}\left(c_{j}-c_{m}\right)},
$$




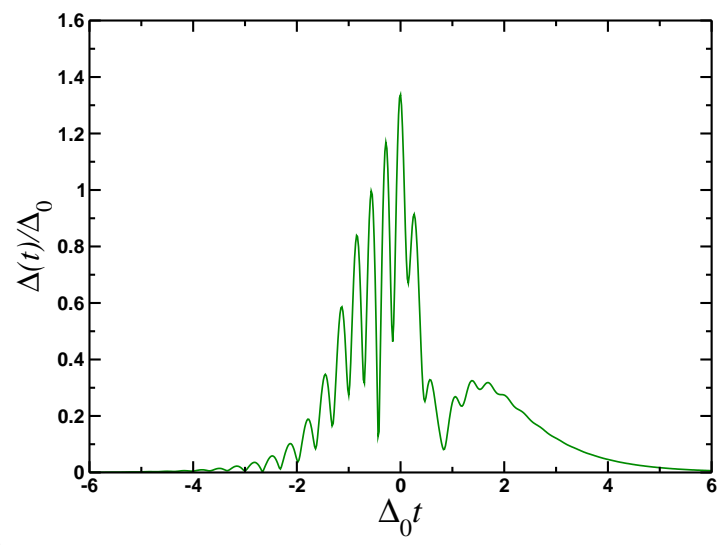

a)

FIG. 3: (color online) 3-normal-soliton solution to the Bogoliubov-de Gennes equations (1) obtained from Eq. (8) with random $c_{j}$ and $\phi_{j} . \Delta_{0}$ is the ground state BCS gap. a) and b) differ only in the values of $\alpha_{j}$. In a) $\alpha_{2}-\alpha_{1}=1.00$ and $\alpha_{3}-\alpha_{2}=1.25$; in b) $\alpha_{2}-\alpha_{1}=10$ and $\alpha_{3}-\alpha_{2}=12$. We see that for large differences between $\alpha_{j}$ in b) the 3-soliton breaks up into a sum of three well separated individual solitons, see Eq. (12), while in a) the same solution but with small differences describes a complicated interference between the three solitons.

The set of $2 k$ complex parameters $c_{m}$ ("frequencies" of the unstable modes) is complex conjugate to itself. Let us order this set so that $c_{k+l}=c_{l}^{*}$ and $\operatorname{Im}\left(c_{l}\right)>0$ for $l=1, \ldots, k$. The constants $A\left(c_{l}\right)$ and $A\left(c_{k+l}\right)$ are related as follows

$$
A\left(c_{l}\right)=e^{\alpha_{l}+i \phi_{l}}, \quad A\left(c_{k+l}\right)=-e^{-\alpha_{l}+i \phi_{l}}
$$

where $\alpha_{l}$ and $\phi_{l}$ are arbitrary real parameters. The single soliton (4) is obtained from Eq. (8) by setting $k=1$ and $c_{1}=\mu+i \gamma$. The 2 -soliton corresponds to $k=2$ and $c_{1,2}=i \gamma_{1,2}$ or $c_{1,2}=i \gamma \pm \mu$ to get Eq. (6) or (77), respectively. See also Fig. 3 for examples of 3-normal-solitons.

At $t \rightarrow \pm \infty$ the $k$-normal-soliton tends to a normal eigenstate that has at least $2 k-1$ discontinuities in the distribution function $n(\epsilon)$. Linearizing the Bogoliubov-de Gennes equations around this state at large negative $t$, one obtains $k$ unstable normal modes that grow as $e^{-2 i c_{l} t}$ for $l=1, \ldots, k$. When the differences between parameters $\alpha_{l}$ are large the $k$-soliton solution (8) breaks up into a sum of $k$ single solitons,

$$
\left|\Delta^{(k)}\left(t,\left\{c_{i}, \alpha_{i}, \phi_{i}\right\}\right)\right| \approx \sum_{i=1}^{k}\left|\Delta^{(1)}\left(t, \operatorname{Im}\left(c_{i}\right), \alpha_{i}\right)\right|
$$

where $\Delta^{(k)}(t)$ denotes the $k$-normal-soliton (8) and $\left|\Delta^{(1)}\left(t, \operatorname{Im}\left(c_{i}\right), \alpha_{i}\right)\right|$ stands for the single soliton (4) with $\gamma=\operatorname{Im}\left(c_{i}\right)$ and $\alpha=\alpha_{i}$. In this case, the plot of $|\Delta(t)|$ shows $k$ well separated peaks (individual solitons) as illustrated in Figs. 2 and 3 . $\operatorname{Im}\left(2 c_{l}\right)$ set the amplitudes and widths of 


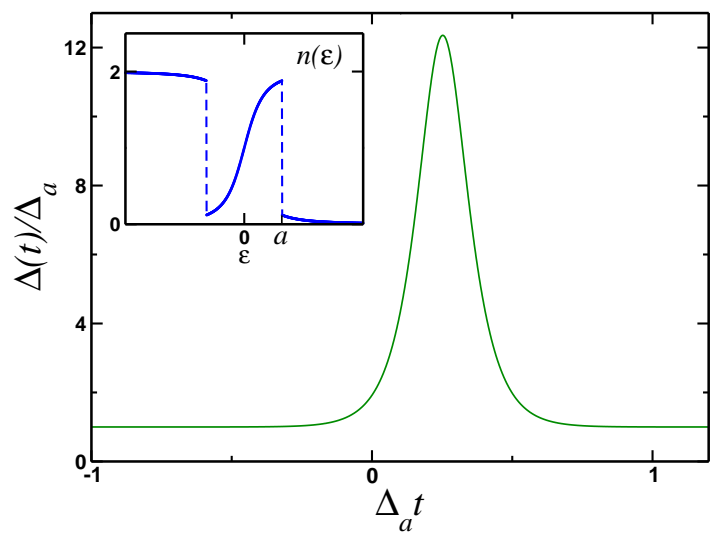

FIG. 4: (color online) $k=1$ anomalous soliton solution to the Bogoliubov-de Gennes equations (11) as given by Eq. (13). At $t \rightarrow \pm \infty$ the system tends to an eigenstate of the BCS Hamiltonian (3) with order parameter $\Delta_{a}$. This eigenstate is characterized by $2 k=2$ jumps at $\epsilon= \pm a$ in the average fermion occupation number $n(\epsilon)$ (inset). $\gamma$ and $\Delta_{a}$ in Eq. (13) are related to $a$ and the ground state gap $\Delta_{0}$ via Eqs. (48) and (50). Here $a=1.47 \Delta_{a}$ and $\Delta_{a}=.09 \Delta_{0}$.

individual solitons, $\operatorname{Re}\left(2 c_{l}\right)$ are the frequencies with which they "rotate" with respect to one another as in Eq. (7), where $\operatorname{Re}\left(c_{1,2}\right)= \pm \mu$, and $\alpha_{l}$ and $\phi_{l}$ determine the separation between the solitons and their relative phases, respectively.

\section{B. Anomalous solitons}

Next, let us summarize the results of Sec. $\mathrm{V}$ for anomalous solitons. A single anomalous soliton has the following form (see also Fig. 4):

$$
\Delta(t)-\Delta_{a}=\frac{2\left(\gamma^{2}-\Delta_{a}^{2}\right)}{\Delta_{a} \pm \gamma \cosh (\lambda t+\alpha)},
$$

where $\lambda=2 \sqrt{\gamma^{2}-\Delta_{a}^{2}}$ and $\alpha$ is an arbitrary real parameter as before. As $t \rightarrow \pm \infty$ the state of the system tends to an anomalous eigenstate with the value of the BCS order parameter equal to $\Delta_{a}$. In this state, all pairs in a certain energy interval around the Fermi level are excited, i.e. $E_{m}=\sqrt{\epsilon_{m}^{2}+\Delta_{a}^{2}}$ for $\left|\epsilon_{m}\right|<a$. As a result, the distribution function $n(\epsilon)$ has two jumps at $\epsilon= \pm a$ (inset in Fig. (4). A linear analysis around this anomalous eigenstate shows a single unstable mode that grows as $e^{\lambda t}$. In general, $2 k$ jumps in the distribution function of a stationary anomalous state give rise to up to $k$ anomalous solitons. Note also that the anomalous soliton (13) generalizes the normal one (44) and turns into it when $\Delta_{a}=0$.

A general $k$-anomalous-soliton can also be constructed within our approach. However, here we 

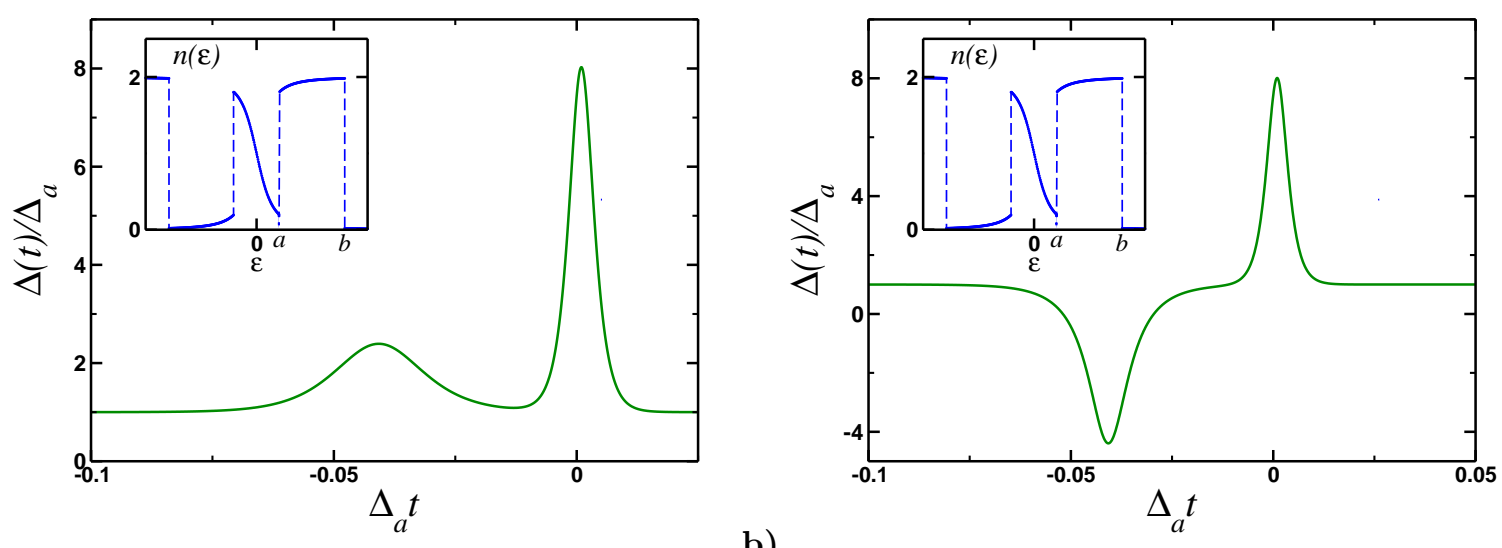

a)

b)

FIG. 5: (color online) $k=2$ anomalous soliton solutions of the Bogoliubov-de Gennes equations (11), see Eqs. (14) and (15). a) corresponds to the choice of signs ++ and b) to -+ in Eq. (15). At $t \rightarrow \pm \infty$ both 2-anomalous-solitons tend to the same stationary state of the mean-field BCS Hamiltonian. In this state, $n(\epsilon)$ has $2 k=4$ discontinuities at $\epsilon= \pm a$ and $\pm b$ (insets) and the anomalous average is equal to $\Delta_{a}$. Parameters $\gamma_{1,2}$ in Eq. (15) and the value of $\Delta_{a}$ are determined by $a, b$, and the ground state gap $\Delta_{0}$, see Eqs. (51) and (52). For the above plots we used $a=.87 \Delta_{a}, b=4.27 \Delta_{a}$, and $16 \Delta_{a}=\Delta_{0}$.

present only an example of a 2-anomalous-soliton solution

$$
\Delta(t)-\Delta_{a}=\frac{h(t)}{2\left[h(t) \ddot{h}(t)-\dot{h}^{2}(t)\right]},
$$

where

$$
h(t)=\frac{\Delta_{a}}{\lambda_{1}^{2} \lambda_{2}^{2}} \pm \frac{\gamma_{1} \cosh \left(\lambda_{1} t+\alpha_{1}\right)}{\lambda_{1}^{2}\left(\lambda_{1}^{2}-\lambda_{2}^{2}\right)} \pm \frac{\gamma_{2} \cosh \left(\lambda_{2} t+\alpha_{2}\right)}{\lambda_{2}^{2}\left(\lambda_{2}^{2}-\lambda_{1}^{2}\right)},
$$

The \pm signs can be chosen independently of each other. As in the case of the 1-anomalous-soliton, at large times the wave function asymptotes to an anomalous stationary state with order parameter $\Delta_{a}$. This state has two unstable modes that grow exponentially with rates $\lambda_{1,2}=2 \sqrt{\gamma_{1,2}^{2}-\Delta_{a}^{2}}$. The graph of the 2-anomalous-soliton solution (14) consists of two peaks or dips depending on the choice of signs in Eq. (15), see Fig. 5. The parameters $\alpha_{1,2}$ take arbitrary real values. Their difference, $\left|\alpha_{1}-\alpha_{2}\right|$, determines the separation between the peaks in time. Similarly to Eq. (12), at large separations the $k$-anomalous-soliton turns into a sum of individual solitons of the form (13)

$$
\Delta^{(k)}\left(t,\left\{\lambda_{i}, \alpha_{i}\right\}\right)-\Delta_{a} \approx \sum_{i=1}^{k}\left[\Delta^{(1)}\left(t, \lambda_{i}, \alpha_{i}\right)-\Delta_{a}\right],
$$

where $\Delta^{(1)}\left(t, \lambda_{i}, \alpha_{i}\right)$ is the single anomalous soliton (13) with $\lambda=\lambda_{i}$ and $\alpha=\alpha_{i}$.

The rest of the paper is organized as follows: in the next section, we review the basic setup of the problem and the tools (Lax vector and separation variables) necessary for deriving solitons. In 
Sec. IV, we perform linear analysis of equations of motion around normal and anomalous stationary states. This section also provides examples of normal and anomalous eigenstates that give rise to one and two normal and anomalous solitons. Sections [V]and V are devoted to a detailed derivation of soliton solutions and a discussion of their main properties.

\section{REVIEW OF THE BASIC SETUP AND RELEVANT PREVIOUS RESULTS}

In this section we discuss the basic setup of the problem and introduce our notation (see Refs. 27, 30 for more details). We also review the properties of the exact solution [29, 30, 33, 34] of the equations of motion needed for obtaining and analyzing the multi-soliton solutions summarized in the previous section.

\section{A. Notations and basic equations}

Here we review the model Hamiltonian and its mean-field equations of motion (1). The latter can be reformulated as equations of motion for classical spins (angular momenta) - this is the form we will be primarily using. We also describe normal and anomalous stationary states in terms of the spin variables.

The dissipationless dynamics of a fermionic superfluid can be modeled by the reduced BCS Hamiltonian [24, 25, 27, 38]

$$
\hat{H}=\sum_{j} \epsilon_{j} \hat{n}_{j}-g \sum_{j, k} \hat{c}_{j \uparrow}^{\dagger} \hat{c}_{j \downarrow}^{\dagger} \hat{c}_{k \downarrow} \hat{c}_{k \uparrow}
$$

where $\hat{n}_{j}=\sum_{\sigma=\downarrow, \uparrow} \hat{c}_{j \sigma}^{\dagger} \hat{c}_{j \sigma}$. This description is valid in the weak coupling regime at times shorter than the energy relaxation time $\tau_{\epsilon}$ and for a system of size $L$ smaller than the BCS coherence length $\xi$. Under these conditions, the BCS order parameter is uniform in space, the interaction matrix elements can be evaluated at the Fermi energy $\epsilon_{F}$ yielding a single coupling constant $g$ that is independent of $j$ and $k$. The summations in Eq. (17) over $j$ and $k$ are restricted to single particle energies $\left|\epsilon_{j}\right|<D$ and $\left|\epsilon_{k}\right|<D$, where $D$ is an ultraviolet cutoff for the pairing interaction. In metallic superconductors $D \simeq \omega_{D}$, where $\omega_{D}$ is the Debye frequency. For atomic fermions $D \simeq \epsilon_{F}$.

The offdiagonal interactions - terms of the form $c_{j \uparrow}^{\dagger} \hat{c}_{l \downarrow}^{\dagger} \hat{c}_{k \downarrow} \hat{c}_{r \uparrow}$ with $l \neq j$ or $r \neq k$ can be neglected, since they are relevant only at times $t>\tau_{\epsilon}$.

The validity of the mean-field approach is rooted in the fact that each pair creation operator $\hat{c}_{j \uparrow}^{\dagger} \hat{c}_{j \downarrow}^{\dagger}$ in Eq. (17) interacts with the collective pairing field $g \sum_{j} \hat{c}_{k_{\downarrow}} \hat{c}_{k \uparrow}$, which is expected to deviate 
little from its quantum mechanical average $\Delta(t)$. For example, the mean-field is known to be exact for the description of the low-energy properties of the Hamiltonian (17) in the limit $\delta / \Delta_{0} \rightarrow 0$ [40, 45, 47], where $\delta=\left\langle\epsilon_{m+1}-\epsilon_{m}\right\rangle$ and $\Delta_{0}$ are the mean spacing between the single particle levels $\epsilon_{m}$ and the ground state gap, respectively. Note that the conditions $\delta \ll \Delta_{0}$ and $L \ll \xi$ are compatible in the weak coupling regime.

We are interested in solving the Heisenberg equations of motion for Hamiltonian (17) to determine the evolution of various correlators, e.g. $\left\langle\hat{n}_{m}(t)\right\rangle,\left\langle\hat{c}_{m \downarrow}(t) \hat{c}_{m \uparrow}(t)\right\rangle$, and $\left\langle\hat{c}_{m \uparrow}^{\dagger}(t) \hat{c}_{m \downarrow}^{\dagger}(t)\right\rangle$. In mean-field approach, we replace the operator $g \sum_{j} \hat{c}_{k \downarrow}(t) \hat{c}_{k \uparrow}(t)$ in the Heisenberg equations with its quantum mechanical average

$$
\Delta(t)=g \sum_{k}\left\langle\hat{c}_{k \downarrow}(t) \hat{c}_{k \uparrow}(t)\right\rangle .
$$

Further, introducing

$$
\begin{aligned}
& 2 s_{m}^{z}=\left\langle\hat{n}_{m}\right\rangle-1, \\
& s_{m}^{-} \equiv s_{m}^{x}-i s_{m}^{y}=\left\langle\hat{c}_{m \downarrow} \hat{c}_{m \uparrow}\right\rangle,
\end{aligned}
$$

we obtain [40]

$$
\dot{\mathbf{s}}_{m}=\mathbf{b}_{m} \times \mathbf{s}_{m}, \quad \mathbf{b}_{m}=\left(-2 \Delta_{x},-2 \Delta_{y}, 2 \epsilon_{m}\right),
$$

where $\Delta_{x}$ and $-\Delta_{y}$ are the real and imaginary parts of $\Delta=g \sum_{m} s_{m}^{-}$. In terms of $F_{m}(t)=$ $2 i s_{m}^{z}$ and $G_{m}(t)=2 i s_{m}^{-}$, Green's functions at coinciding times, Eqs. (20) are well-known Gorkov equations [24, 46]. The above procedure leading to Gorkov equations is essentially equivalent to taking the time-dependent wave function of the system to have the product form (2) at all times. Then, the Schrödinger equation takes the form of the Bogoliubov-de Gennes equations (11), which are in turn equivalent to Eq. (20) with

$$
2 s_{m}^{z}=\left|V_{m}\right|^{2}-\left|U_{m}\right|^{2}, \quad s_{m}^{-}=U_{m} V_{m}^{*}
$$

Equations of motion (20) are Hamilton's equations for the following classical spin (interacting angular momentum) model:

$$
H_{c l}=\sum_{j=1}^{n} 2 \epsilon_{j} s_{j}^{z}-g \sum_{j, k=1}^{n} s_{j}^{+} s_{k}^{-},
$$

with the usual angular momentum Poisson brackets $\left\{s_{j}^{x}, s_{j}^{y}\right\}=-s_{j}^{z}$ etc. The summations in Eq. (22) are restricted to the subspace of unblocked (with occupation numbers $n_{j}=0,2$ ) single fermion 
levels $\epsilon_{j}$. The Hamiltonian (17) does not have matrix elements connecting the unblocked levels to blocked ones $\left(n_{j}=1\right)$. The latter are decoupled and their occupation numbers are conserved by the evolution. Note also that Eq. (11) conserves the norm $\left|U_{m}\right|^{2}+\left|V_{m}\right|^{2}=1$ and therefore the length of the spins is fixed $\left|\mathbf{s}_{m}\right|=1 / 2$.

The normal and anomalous eigenstates of the BCS Hamiltonian discussed in the Introduction correspond to equilibrium spin configurations where each spin $\mathbf{s}_{m}$ is either parallel or antiparallel to the field $\mathbf{b}_{m}[40]$. According to Eq. (21), every such arrangement of spins uniquely determines an eigenstate of the form (2) and vice versa. The anomalous eigenstates yield

$$
2 s_{m}^{z}=-\frac{e_{m} \epsilon_{m}}{\sqrt{\epsilon_{m}^{2}+\Delta_{a}^{2}}}, \quad 2 s_{m}^{x}=-\frac{e_{m} \Delta_{a}}{\sqrt{\epsilon_{m}^{2}+\Delta_{a}^{2}}}, \quad e_{m}= \pm 1
$$

where the $x$ axis has been chosen so that the stationary value of the order parameter, $\Delta_{a}$, is real. The factor $e_{m}=-1$ if the spin is parallel to the field and $e_{m}=1$ otherwise. The self-consistency condition $\Delta_{a}=g \sum_{m} s_{m}^{x}$ reads

$$
\sum_{m} \frac{e_{m}}{\sqrt{\epsilon_{m}^{2}+\Delta_{a}^{2}}}=\frac{2}{g}
$$

This is the BCS gap equation, which determines the value of $\Delta_{a}$ in the anomalous state. The configuration of spins with all $e_{m}=1$ is equivalent to the BCS ground state. In this case $\Delta_{a}=\Delta_{0}$ - the ground state gap - and Eq. (24) becomes in the continuum limit

$$
\int_{0}^{D} \frac{d \epsilon}{\sqrt{\epsilon^{2}+\Delta_{0}^{2}}}=\frac{2}{\lambda} \quad \lambda=g \nu_{F} V \equiv \frac{g}{\delta},
$$

where $\nu_{F}$ is the density of states at the Fermi level and $V$ is the volume of the system. In Eq. (25) and throughout this paper we assume the weak coupling regime $\Delta_{0} \ll D$ and a constant density of $\epsilon_{j}, \nu(\epsilon)=\nu_{F}$, in the continuum limit. A non-constant density of states modifies the value of $\Delta_{0}$ determined from Eq. (25) but will not affect any other equations derived in the rest of the paper. As we will see, these equations are confined to energies of order $\Delta_{0}$, while the density of states varies on an energy scale of order $D$ or larger. Using $\Delta_{0} \ll D$, we obtain from Eq. (25)

$$
\Delta_{0}=2 D e^{-1 / \lambda}
$$

The configuration with only one flipped spin, $e_{k}=-1$ and $e_{m \neq k}=1$, corresponds to an excited state - it contains an excited pair and has energy $2 \sqrt{\epsilon_{k}^{2}+\Delta_{0}^{2}}$ relative to the ground state. Similarly, having two spins parallel to the field is equivalent to an eigenstate with two excited pairs etc.

Normal eigenstates are spin arrangements where each spin is along $z$ axis, i.e.

$$
2 s_{m}^{z}= \pm 1 \equiv l_{m}, \quad s_{m}^{-}=0 .
$$


They are also equilibria of the classical Hamiltonian (22) according to Eq. (201). The Fermi ground state corresponds to $l_{m}=-\operatorname{sgn} \epsilon_{m}$, while other normal eigenstates can be obtained from this state by moving pairs of fermions from levels below the Fermi energy to levels above it and flipping the corresponding spins.

\section{B. General properties of the dynamics}

In this subsection we introduce the Lax vector construction [30, 33], which plays a central role in analyzing the dynamics of the BCS Hamiltonian. We also define the separation variables and describe the general features of the dynamics.

The dynamics of the classical Hamiltonian (22) or, equivalently, Eqs. (20) and (11) turn out to be integrable. A convenient tool for their analysis is the Lax vector defined as

$$
\mathbf{L}(u)=-\frac{\hat{\mathbf{z}}}{g}+\sum_{m=1}^{n} \frac{\mathbf{s}_{m}}{u-\epsilon_{m}},
$$

where $u$ is a complex parameter, $\hat{\mathbf{z}}$ is a unit vector along $z$ axis, and $n$ is the total number of spins. The length of this vector is conserved by Eqs. (20) for any $u$, i.e.

$$
\frac{d \mathbf{L}^{2}(u)}{d t}=0
$$

For this reason $\mathbf{L}^{2}(u)$ can be viewed as the generator of the integrals of motion [30] for Eqs. (20). For example, its zeroes are conserved and constitute a set of independent integrals. Another possible choice for the integrals is e.g. the set of the residues of $\mathbf{L}^{2}(u)$ at the poles at $u=\epsilon_{m}$. Note that

$$
\mathbf{L}^{2}(u)=\frac{Q_{2 n}(u)}{g^{2} \prod_{j}\left(u-\epsilon_{j}\right)^{2}},
$$

where $Q_{2 n}(u)$ is a (spectral) polynomial of order $2 n$. We also have

$$
\mathbf{L}^{2}(u)=L_{z}^{2}(u)+L_{-}(u) L_{+}(u)
$$

where $L_{-}(u)=L_{x}(u)-i L_{y}(u)$ and $L_{x, y, z}$ are the components of the Lax vector $\mathbf{L}(u)$.

To obtain solitons, we need to introduce new dynamical variables $u_{m}$ [48, 49] in which Eqs. (20) separate and can be integrated. The separation variables are defined in terms of the "old" dynamical variables $\mathbf{s}_{j}$ as solutions of the following equation:

$$
L_{-}\left(u_{m}\right)=\sum_{j=1}^{n} \frac{s_{j}^{-}}{u_{m}-\epsilon_{j}}=0 .
$$


This equation has $n-1$ solutions since, when $L_{-}(u)$ is brought to a common denominator, its numerator is a polynomial of order $n-1$. Consequently, there are $n-1$ separation variables $u_{m}$. Eq. (32) can be inverted to obtain the spins in terms of the separation variables as follows

$$
s_{j}^{-}=J_{-} \frac{\prod_{k}\left(\epsilon_{j}-u_{k}\right)}{\prod_{k \neq j}\left(\epsilon_{j}-\epsilon_{k}\right)},
$$

where $J_{-}=J_{x}-i J_{y}$ as usual and $\mathbf{J}=\sum_{j} \mathbf{s}_{j}$ is the total classical spin.

In terms of the separation variables Eqs. (20) read

$$
\begin{aligned}
& \dot{u}_{j}=\frac{2 i \sqrt{Q_{2 n}\left(u_{j}\right)}}{\prod_{m \neq j}\left(u_{j}-u_{m}\right)}, \quad j=1, \ldots, n-1, \\
& \dot{J}_{-}=-2 i J_{-}\left(\sum_{j=1}^{n} \epsilon_{j}+\frac{g J_{z}}{2}-\sum_{m=1}^{n-1} u_{m}\right) .
\end{aligned}
$$

An important observation[34] is that main properties of the dynamics can be effectively discerned by analyzing the zeros of $\mathbf{L}^{2}(u)$. According to Eq. (30), these are the roots of the spectral polynomial $Q_{2 n}(u)$ and we will often refer to their configuration in the complex plane as to the root diagram of $\mathbf{L}^{2}(u)$. Since $Q_{2 n}(u)$ is positively defined, it has $n$ pairs of complex conjugate roots. For generic initial conditions all $2 n$ roots are distinct. In this case the dynamics of the system is quasiperiodic with $n$ incommensurate frequencies and any dynamical quantity, e.g. the order parameter $\Delta(t)=g J_{-}(t)$, typically contains all $n$ frequencies.

Significant simplifications occur when some roots are degenerate [29, 33]. It is important to distinguish between real and complex double roots. Note that any real root of $Q_{2 n}(u)$ is automatically a double root (zero) because $Q_{2 n}(u)$ is positively defined. A real zero $c$ of $\mathbf{L}^{2}(u)$ must also be a zero of all three components of $\mathbf{L}(u)[50]$. Further, note from Eq. (32) that one of the separation variables must coincide with $c$. In other words, it must be time-independent as it is "frozen" into the real root $c$. Eq. (34) shows that this is an allowed solution of the equations of motion for the separation variables. This freezing of a separation variable can be translated into a genuine reduction of the number of degrees of freedom by one so that the dynamics of the Hamiltonian (22) with $n$ spins reduces to that of the same Hamiltonian but with $n-1$ spins. In general, $n-m$ real zeros (or equivalently $2 m$ complex zeros) mean a reduction of the dynamics to that of $2 m$ effective spins, see Ref. 29 and 33 for details. Below we will often encounter a situation when $\mathbf{L}^{2}(u)$ has a number of real zeros and consequently a number of separation variables are frozen. The remaining variables we call unfrozen.

Let $u_{n-1}=c$ be the separation variable frozen into the double zero of $Q_{2 n}(u)$. Consider Eq. (34) for $j \neq n-1$. Both the numerator and the denominator of the right hand side contain a factor $u-c$, 
which cancels lowering the order of the polynomial under the square root by two. Suppose $Q_{2 n}(u)$ has $n-2 k$ double real zeros. Then, there are $2 k-1$ unfrozen separation variables $u_{1}, \ldots, u_{2 k-1}$. For these variables Eq. (34) can be brought to the following form[29] with the help of Eq. (301):

$$
\sum_{j=1}^{2 k-1} \frac{u_{j}^{l} d u_{j}}{\prod_{m}\left(u_{j}-\epsilon_{m}\right) \sqrt{\widetilde{\mathbf{L}}^{2}\left(u_{j}\right)}}=2 i g d t \delta_{l, 2 k-2}, \quad l=0, \ldots, 2 k-2,
$$

where $\widetilde{\mathbf{L}}^{2}(u)$ is obtained from $\mathbf{L}^{2}(u)$ by removing all real zeros $c_{m}$, i.e.

$$
\widetilde{\mathbf{L}}^{2}(u)=\frac{\mathbf{L}^{2}(u)}{\prod_{m}\left(u-c_{m}\right)^{2}} .
$$

\section{LINEAR ANALYSIS AROUND STATIONARY STATES}

In this section, we analyze equations of motion linearized in the vicinity of normal and anomalous stationary states. We show that the separation variables $u_{j}$ are the normal modes of the linearized problem. Some of the stationary states are unstable. As we will see in the next section, the corresponding normal modes become solitons in the nonlinear regime.

\section{A. Frequencies of oscillations around stationary states}

Here we show that the frequencies of small oscillations around normal and anomalous states are determined by the zeros of $\mathbf{L}^{2}(u)$, see also Ref. $\underline{34}$. When one of the frequencies is complex, the state is unstable and the corresponding mode grows exponentially.

The linear analysis of equations of motion (20) around stationary states greatly simplifies in terms of separation variables. According to Eq. (34), stationary positions of $u_{j}$ are the roots of the polynomial $Q_{2 n}(u)$ (or equivalently the zeros of $\mathbf{L}^{2}(u)$, see Eq. (30)). Let us determine the form of $\mathbf{L}^{2}(u)$ in the stationary states. Consider first the anomalous states (23). Using Eqs. (23), (28) , and (24), we obtain

$$
\mathbf{L}(u)=\left(\Delta_{a} \hat{\mathbf{x}}-u \hat{\mathbf{z}}\right) L_{s}(u), \quad \mathbf{L}^{2}(u)=\left(u^{2}+\Delta_{a}^{2}\right) L_{s}^{2}(u),
$$

where $\hat{\mathbf{x}}$ is a unit vector along $x$ axis and

$$
L_{s}(u)=\sum_{m=1}^{n} \frac{e_{m}}{2\left(u-\epsilon_{m}\right) \sqrt{\epsilon_{m}^{2}+\Delta_{a}^{2}}}, \quad e_{m}= \pm 1 .
$$

Note that when the right hand side of Eq. (38) is brought to a common denominator, the numerator is a polynomial of order $n-1$. Therefore, $\mathbf{L}^{2}(u)$ and consequently $Q_{2 n}(u)$ have $n-1$ double zeros 
$c_{r}$ - the solutions of the equation $L_{s}\left(c_{r}\right)=0$. In addition, we see from Eq. (37) that there are two roots $u= \pm i \Delta_{a}$, i.e.

$$
Q_{2 n}(u)=\left(u^{2}+\Delta_{a}^{2}\right) \prod_{r=1}^{n-1}\left(u-c_{r}\right)^{2}
$$

When the spins $\mathbf{s}_{j}$ deviate from their equilibrium positions (23), the roots $c_{r}$ of polynomial $Q_{2 n}(u)$ shift to $c_{r}+\delta c_{r}[$ [1] $]$. Linearizing Eq. (34) in deviations $\delta c_{r}=a_{r}+i b_{r}$ and $\delta u_{r}=u_{r}-c_{r}-a_{r}$ around the stationary positions $u_{r}=c_{r}$ and using Eq. (39), we obtain

$$
\delta \dot{u}_{r}=2 i \sqrt{c_{r}^{2}+\Delta_{a}^{2}} \sqrt{\left(\delta u_{r}\right)^{2}+b_{r}^{2}}
$$

with a solution $\delta u_{r}=b_{r} \sin \left[\omega_{r}\left(t-t_{0}\right)\right]$, where $\omega_{r}=2 \sqrt{c_{r}^{2}+\Delta_{a}^{2}}$. Linearizing Eq. (33), one derives the spin variables in terms of $\delta u_{r}$. At this point we are interested only in the frequencies $\omega_{r}$.

We conclude that the separation variables are indeed the normal modes of the linearized problem (since they contain a single frequency). The frequencies of small oscillations around anomalous stationary states are related to the double zeros $c_{r}$ of $\mathbf{L}^{2}(u)$ as $\omega_{r}=2 \sqrt{c_{r}^{2}+\Delta_{a}^{2}}$. If any of $\omega_{r}$ has an imaginary part, the stationary state is unstable.

Next, consider linear analysis around normal eigenstates (27). In this case all spins are along $z$ axis. It follows from Eqs. (27) and (28) that $\mathbf{L}(u)=L_{n}(u) \hat{\mathbf{z}}$, where

$$
L_{n}(u)=-\frac{1}{g}+\sum_{j=1}^{n} \frac{l_{j}}{2\left(u-\epsilon_{j}\right)}, \quad l_{j}= \pm 1 .
$$

We see that all zeros $c_{r}$ of $\mathbf{L}^{2}(u)=L_{n}^{2}(u)$ are double zeros. There are $n$ of them as the numerator of $L_{n}(u)$ is a polynomial of order $n$, i.e. $Q_{2 n}(u)=\prod_{r=1}^{n}\left(u-c_{r}\right)^{2}$. As before, the stationary positions of separation variables are $u_{r}=c_{r}$. Note however that there are only $n-1$ separation variables, so one of the $n$ zeros $c_{r}$ must remain vacant.

Next, we show that the frequencies of small oscillations around a normal stationary state are $\omega_{r}=2 c_{r}$. When one of the zeros $c_{r}$ is complex, the oscillatory behavior is replaced with an exponential growth, i.e. the stationary state is unstable. Note that for small deviations from a normal state the $x y$ components of the total spin $\mathbf{J}$ are small. Therefore, in linear approximation we can set the separation variables $u_{j}$ to their equilibrium values in Eqs. (33) and (35), $u_{j}=c_{j}$, i.e. only $J_{-}$is time-dependent. As mentioned above, $L_{n}(u)$ has a vacant zero (say $c_{r}$ ) which does not correspond to any separation variable. Eq. (35) yields

$$
-\frac{d\left(\ln J_{-}\right)}{2 i d t}=\left[\sum_{j=1}^{n} \epsilon_{j}+\frac{g J_{z}}{2}-\sum_{m=1}^{n} c_{m}\right]+c_{r}=c_{r},
$$


where we used the fact that the contribution in square brackets vanishes. This can be seen by observing that, since $c_{m}$ are the zeros of $L_{n}(u)$, Eq. (41) can be written as

$$
L_{n}(u)=-\frac{1}{g} \frac{\prod_{m}\left(u-c_{m}\right)}{\prod_{j}\left(u-\epsilon_{j}\right)} .
$$

Expanding the right hand sides of Eqs. (43) and (41) in $1 / u$, matching the coefficients at $1 / u$, and using $2 J_{z}=\sum_{j} l_{j}$ (this follows from Eq. (27)), we see that the sum of terms in square brackets in Eq. (42) is indeed zero. It follows that $J_{-} \propto e^{-2 i c_{r} t}$ and from Eq. (33) we also derive $s_{j}^{-} \propto e^{-2 i c_{r} t}$. Thus, the frequencies of oscillations around normal stationary states are $\omega_{r}=2 c_{r}$.

\section{B. Examples of root diagrams of $\mathbf{L}^{2}(u)$}

Here we provide examples of root diagrams - configurations of solutions of the equation $\mathbf{L}^{2}(u)=$ 0 in the plane of complex $u$, see Ref. $\underline{34}$ for more examples. We saw that the zeros of $\mathbf{L}^{2}(u)$ evaluated in stationary states determine the frequencies of oscillations around them. Moreover, the most important features of the dynamics, e.g. the behavior of $\Delta(t)$ at large times, can be

predicted by inspecting the root diagram [34], see also the discussion below Eq. (35). Similarly, we will see that the root diagram determines the number and properties of solitons corresponding to a given stationary state.

For simplicity, we assume particle-hole symmetry, i.e. the single fermion energies $\left\{\epsilon_{m}\right\}$ are symmetric with respect to zero (Fermi level). According to Eq. (23), this means

$$
\begin{aligned}
& s^{x}\left(\epsilon_{m}\right)=s^{x}\left(-\epsilon_{m}\right), \quad s^{z}\left(\epsilon_{m}\right)=-s^{z}\left(-\epsilon_{m}\right), \\
& s^{y}\left(\epsilon_{m}\right)=-s^{y}\left(-\epsilon_{m}\right),
\end{aligned}
$$

where $\mathbf{s}_{m} \equiv \mathbf{s}\left(\epsilon_{m}\right)$. These relations can also be derived from Eq. (19) using particle-hole transformation for fermion creation and annihilation operators $\hat{c}_{\sigma}\left(-\epsilon_{m}\right) \leftrightarrow \hat{c}_{\sigma}^{\dagger}\left(\epsilon_{m}\right)$. Note that relations (44) are preserved by equations of motion (20) and also imply $\Delta_{y}(t)=0$.

\section{BCS ground state}

As discussed below Eq. (24), the BCS ground state corresponds to $e_{m}=1$. We note from Eq. (23) that spin components $s^{x}\left(\epsilon_{m}\right)$ and $s^{z}\left(\epsilon_{m}\right)$ in this state are continuous functions of single particle energy $\epsilon_{m}$. It follows from Eqs. (37) and (38) that $\mathbf{L}^{2}(u)=0$ has two single roots at $u= \pm i \Delta_{0}$, see Fig. 6, and $n-1$ double roots $c_{k}$ that are the solutions of the following equation:

$$
L_{s}(u)=\sum_{m=1}^{n} \frac{1}{2\left(u-\epsilon_{m}\right) \sqrt{\epsilon_{m}^{2}+\Delta_{0}^{2}}}=0 .
$$




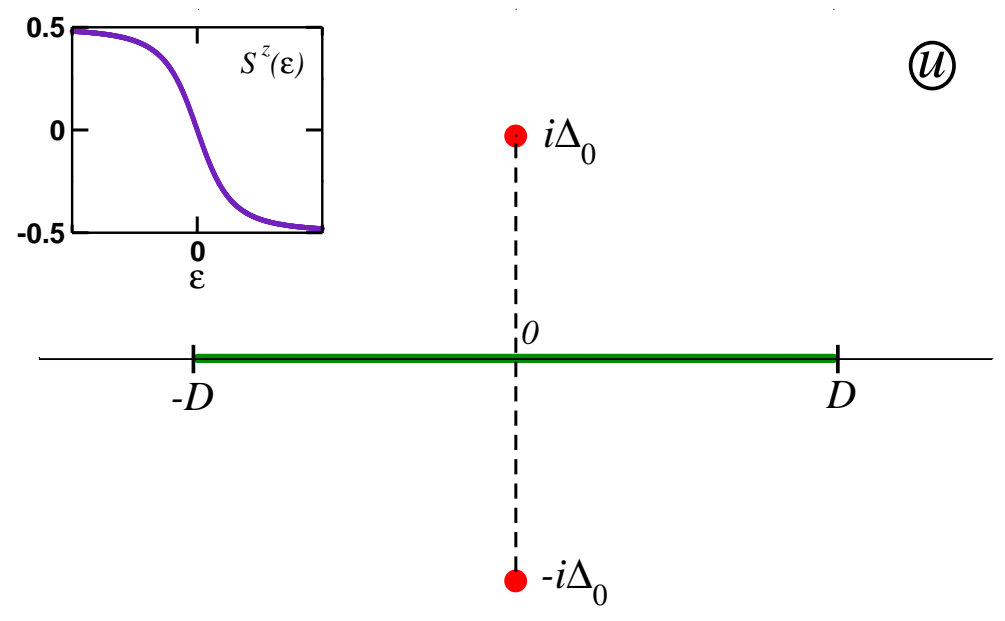

FIG. 6: (color online) The roots of $\mathbf{L}^{2}(u)=0$ (the root diagram) for the BCS ground state in the complex $u$ plane. There is a line of double real roots (zeros of $\mathbf{L}^{2}(u)$ ) from $-D$ to $D$, where $D$ is the high-energy cutoff on the single-fermion states participating in the BCS Hamiltonian (17). In addition, there are two imaginary single zeros $\pm i \Delta_{0}$, where $\Delta_{0}$ is the ground state gap. Frequencies $\omega$ of small oscillations around the ground state are related to the zeros $c$ as $\omega=\sqrt{c^{2}+\Delta_{0}^{2}}$, see Sec. IIIB 1, We have $\omega(\epsilon)=\sqrt{\epsilon^{2}+\Delta_{0}^{2}}$, where $-D \leq \epsilon \leq D$, and $\omega=0$. The inset shows the spin component $s^{z}(\epsilon)$ in the ground state. Since it has no discontinuities, there are no complex double zeros.

All $n-1$ solutions are real. This can be seen by noting that $L_{s}(u)$ changes sign between consecutive $\epsilon_{m}$. Indeed, let $\epsilon_{m}$ be ordered so that $\epsilon_{1}<\epsilon_{2}<\cdots<\epsilon_{n}$. Since $L_{s}(u) \rightarrow+\infty$ as $u \rightarrow \epsilon_{m}^{+}$and $L_{s}(u) \rightarrow-\infty$ as $u \rightarrow \epsilon_{m+1}^{-}$, there is a point $u=c_{m}$ in the interval $\left(\epsilon_{m}, \epsilon_{m+1}\right)$ where $L_{s}\left(c_{m}\right)=0$. According to the previous subsection, this yields a frequency $\omega_{m}=2 \sqrt{c_{m}^{2}+\Delta_{0}^{2}}$ of small oscillations around the BCS ground state. In the continuum limit, when level spacings $\epsilon_{m+1}-\epsilon_{m}$ tend to zero, we have $c_{m} \approx \epsilon_{m}$ and $\omega_{m} \approx 2 \sqrt{\epsilon_{m}^{2}+\Delta_{0}^{2}}$. In this limit, $c_{m}$ densely fill the interval from $-D$ to $D=\max \left|\epsilon_{m}\right|$, i.e. $\mathbf{L}^{2}(u)$ has a line of double roots as shown in Fig. 6. Frequencies $\omega_{m}$ are also the energies of excited pairs - excitations obtained by flipping the spin $\mathbf{s}_{m}$ from its ground state position antiparallel to the field $\mathbf{b}_{m}=\left(-2 \Delta_{0}, 0, \epsilon_{m}\right)$ to an equilibrium position parallel to the field $\mathbf{b}_{m}$, see Ref. 40 for a discussion of this relationship between the frequencies and the excitation spectrum.

\section{Excited anomalous states}

Next, consider two examples of anomalous stationary states obtained from the BCS ground state by flipping spins in certain energy intervals. 


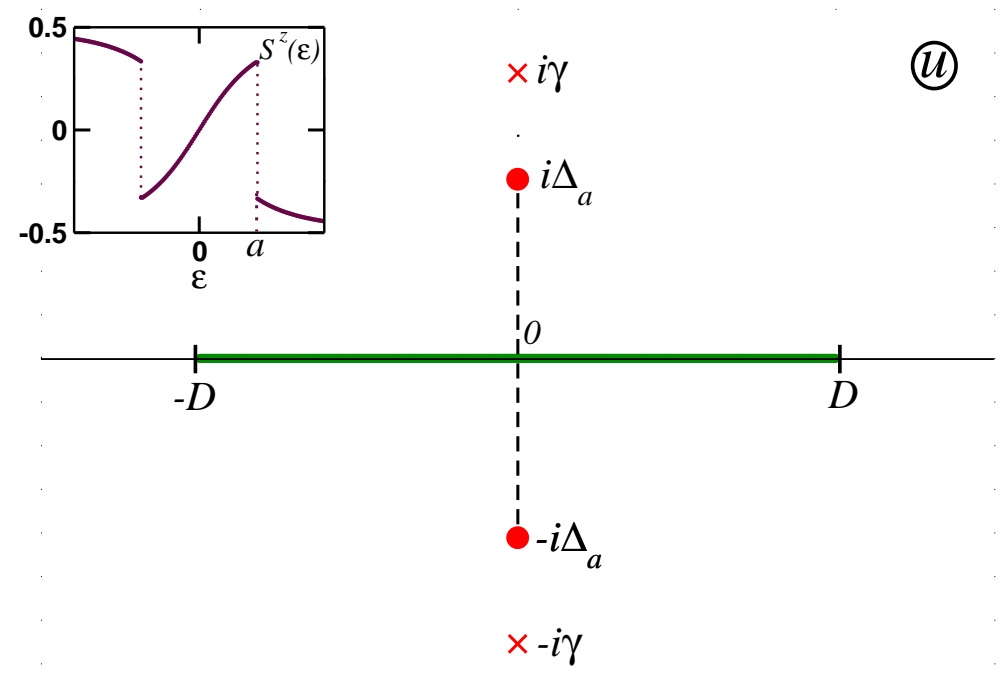

FIG. 7: (color online) Zeros of $\mathbf{L}^{2}(u)$ for an excited anomalous state in the complex $u$ plane. This anomalous state has $2 k=2$ jumps at $\epsilon= \pm a$ in the spin component $s^{z}(\epsilon)$ (inset) leading to $2 k$ double imaginary zeros $\pm i \gamma$. In addition, $\mathbf{L}^{2}(u)$ has a line of real double zeros from $-D$ to $D$ and single zeros $\pm i \Delta_{a}$, where $\Delta_{a}$ is the value of the order parameter in this state. In the present case, there is a single $(k=1)$ unstable mode that grows with the rate $\sqrt{\gamma^{2}-\Delta_{a}^{2}}$ giving rise to a single anomalous soliton shown in Fig. 4, see Sec. IIIB2,

Example 1

First, let spins in the interval $(-a, a)$ be flipped, i.e. $e_{m}=\operatorname{sgn}\left(\left|\epsilon_{m}\right|-a\right)$. This means that the Cooper pairs in this energy interval are excited [38, 40]. Eq. (23) implies that spin components $s^{x}(\epsilon)$ and $s^{z}(\epsilon)$ are discontinuous at $\epsilon= \pm a$ (see the inset in Fig 7). As before $\mathbf{L}^{2}(u)$ has two single zeros at $u= \pm i \Delta_{a}$ and $n-1$ double zeros $c_{k}$ that are the solutions of $L_{s}\left(c_{k}\right)=0$, where $L_{s}(u)$ is given by Eq. (38). The difference is that in this case two of $c_{k}$ can be imaginary. Suppose $\epsilon_{m_{1}}<-a<\epsilon_{m_{1}+1}$ and $\epsilon_{m_{2}}<a<\epsilon_{m_{2}+1}$. Then, $e_{m_{1}}=1$ while $e_{m_{1}+1}=-1$ and similarly for $m_{2}$ and we are no longer guaranteed real zeros of $L_{s}(u)$ in intervals $\left(\epsilon_{m_{1}}, \epsilon_{m_{1}+1}\right)$ and $\left(\epsilon_{m_{2}}, \epsilon_{m_{2}+1}\right)$ as in the BCS ground state. Instead, $L_{s}(u)$ can acquire two complex conjugate zeros. In the particle-hole symmetric case $L_{s}(-u)=-L_{s}(u)$, which implies that these zeros must be purely imaginary as in Fig. 7. The remaining $n-3$ double zeros of $\mathbf{L}^{2}(u)$ are real and lie between consecutive $\epsilon_{j}$. In the continuum limit, they merge into a continuous line of double zeros between $-D$ and $D$ as in the ground state.

To determine the two imaginary zeros $c= \pm i \gamma$ in the continuum limit, we rewrite the equation 
$L_{s}(u)=0$ in the form

$$
\int_{0}^{\infty} \frac{\operatorname{sgn}(\epsilon-a) d \epsilon}{\left(\epsilon^{2}+\gamma^{2}\right) \sqrt{\epsilon^{2}+\Delta_{a}^{2}}}=0
$$

where we used $L_{s}(-u)=-L_{s}(u)$ and took the ultraviolet cutoff $D$ to infinity. In terms of

$$
F(\epsilon)=\int \frac{d \epsilon}{\left(\epsilon^{2}+\gamma^{2}\right) \sqrt{\epsilon^{2}+\Delta_{a}^{2}}}=\frac{1}{2 \gamma \sqrt{\gamma^{2}-\Delta_{a}^{2}}} \ln \left[\frac{\gamma \sqrt{\epsilon^{2}+\Delta_{a}^{2}}+\epsilon \sqrt{\gamma^{2}-\Delta_{a}^{2}}}{\gamma \sqrt{\epsilon^{2}+\Delta_{a}^{2}}-\epsilon \sqrt{\gamma^{2}-\Delta_{a}^{2}}}\right],
$$

Eq. (46)) reads $F(+\infty)=2 F(a)$. This equation has a unique positive solution

$$
\gamma=\left(a+\sqrt{a^{2}+\Delta_{a}^{2}}\right) \frac{a}{\Delta_{a}}
$$

Note however that the gap equation (24) has solutions only for sufficiently small $a$. To see this, we write down Eq. (24) for the order parameter $\Delta_{a}$ in the anomalous state where $e_{m}=\operatorname{sgn}\left(\left|\epsilon_{m}\right|-a\right)$ and for the gap $\Delta_{0}$ in the BCS ground state where $e_{m}=1$. Equating the left hand sides of the two equations, we obtain

$$
\int_{0}^{D} \frac{d \epsilon}{\sqrt{\epsilon^{2}+\Delta_{0}^{2}}}=\int_{0}^{D} \frac{\operatorname{sgn}(\epsilon-a) d \epsilon}{\sqrt{\epsilon^{2}+\Delta_{a}^{2}}} .
$$

In the $D \rightarrow \infty$ limit this equation yields

$$
\Delta_{a}^{3}-2 \Delta_{0} \Delta_{a}^{2}+\Delta_{0}^{2} \Delta_{a}-4 a^{2}=0
$$

together with the condition $\Delta_{a}<\Delta_{0}$.

The analysis of Eq. (50) shows that there are two solutions $\Delta_{a}<\Delta_{0}$ provided $3 \sqrt{3} a \leq \Delta_{0}$ and no solutions otherwise. Interestingly, for one of the solutions $\gamma \leq \Delta_{a}$, while for the other $\gamma \geq \Delta_{a}$. Which solution do we choose? Note that the quantum Hamiltonian (17) has $2^{n}$ unblocked states. Correspondingly, there are $2^{n}$ choices of $e_{m}= \pm 1$. More than one solution for a given selection of $e_{m}$ means that we have more states in the mean-field than there are eigenstates of the original quantum Hamiltonian. It is natural to expect that among the two solutions for $\Delta_{a}$ the one that yields a stable anomalous state corresponds to the quantum eigenstate. We have shown above that frequencies of small oscillations around anomalous stationary states are related to the zeros $c_{k}$ as $\omega_{k}=\sqrt{c_{k}^{2}+\Delta_{a}^{2}}$. For the zeros $\pm i \gamma$ we have $\omega_{\gamma}=i \sqrt{\gamma^{2}-\Delta_{a}^{2}}$. We see that for $\gamma>\Delta_{a}$ the frequency is imaginary and the corresponding normal mode grows exponentially. Therefore, the solution $\Delta_{a}<\gamma$ yields an unstable anomalous state, while for $\Delta_{a}>\gamma$ we get a stable state. Both states however can play an important role in the description of the dynamical problem (11). 


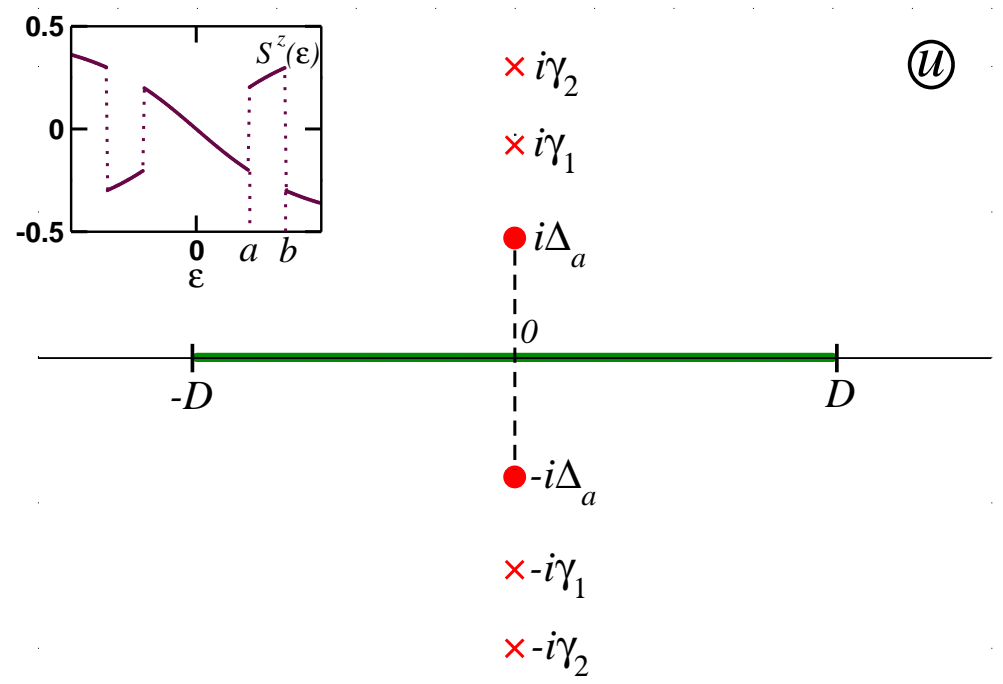

FIG. 8: (color online) Zeros of $\mathbf{L}^{2}(u)$ for an excited anomalous state in the complex $u$ plane. This anomalous state has $2 k=4$ discontinuities at $\epsilon= \pm a$ and $\pm b$ in the spin component $s^{z}(\epsilon)$ (inset) leading to $2 k$ double imaginary zeros $\pm i \gamma_{1,2}$. In addition, $\mathbf{L}^{2}(u)$ has a line of real double zeros from $-D$ to $D$ and single zeros $\pm i \Delta_{a}$, where $\Delta_{a}$ is the value of the order parameter in this state. In the present case, there are $k=2$ unstable modes that grow with rates $\sqrt{\gamma_{1,2}^{2}-\Delta_{a}^{2}}$ giving rise to 2-anomalous-solitons shown in Fig. 5 , see Sec. ஹI B 2.

\section{Example 2}

A more involved example of an anomalous state is obtained by flipping spins in two energy intervals, e.g. in intervals $(-b,-a)$ and $(a, b)$ symmetric with respect to the Fermi level. This implies $e_{m}=\operatorname{sgn}\left(\left|\epsilon_{m}\right|-a\right)\left(\left|\epsilon_{m}\right|-b\right)$. Now the spin components have four discontinuities at $\epsilon= \pm a$ and $\epsilon= \pm b$ (inset in Fig. 8). Correspondingly, $\mathbf{L}^{2}(u)$ can have four complex double zeros $\pm i \gamma_{1,2}$ as in Fig. 8 in addition to two single zeros at $u= \pm i \Delta_{a}$ and $n-5$ real zeros on the line from $-D$ to $D$. This follows in a manner similar to the above analysis of the state with $e_{m}=\operatorname{sgn}\left(\left|\epsilon_{m}\right|-a\right)$. In general, $2 k$ discontinuities in spin components in an anomalous stationary state can lead to $2 k$ complex double roots.

To determine the complex zeros in the continuum limit, we repeat the procedure that lead to Eq. (48). Now we derive $2 F(b)-2 F(a)=F(+\infty)$, where $F(\epsilon)$ is given by expression (47). This equation has solutions $\pm i \gamma_{1,2}$, where

$$
4 \frac{\gamma_{1,2}}{\Delta_{a}}=x y-1 \pm \sqrt{(x y+1)^{2}-4(x+y-1)}
$$

$x \Delta_{a}^{2}=\left(\sqrt{a^{2}+\Delta_{a}^{2}}-a\right)^{2}$, and $y \Delta_{a}^{2}=\left(\sqrt{b^{2}+\Delta_{a}^{2}}+b\right)^{2}$. The gap equation (24) in terms of $x$ and 
$y$ takes the form

$$
x y=\frac{\Delta_{0}}{\Delta_{a}}
$$

where $\Delta_{0}$ is the ground state gap. Using these equations, it is not difficult to select $a$ and $b$ so that $\gamma_{1,2}$ are real and $\gamma_{2}>\gamma_{1}>\Delta_{a}$ as shown in Fig. 8. This is the choice we will need in Sec. VB.

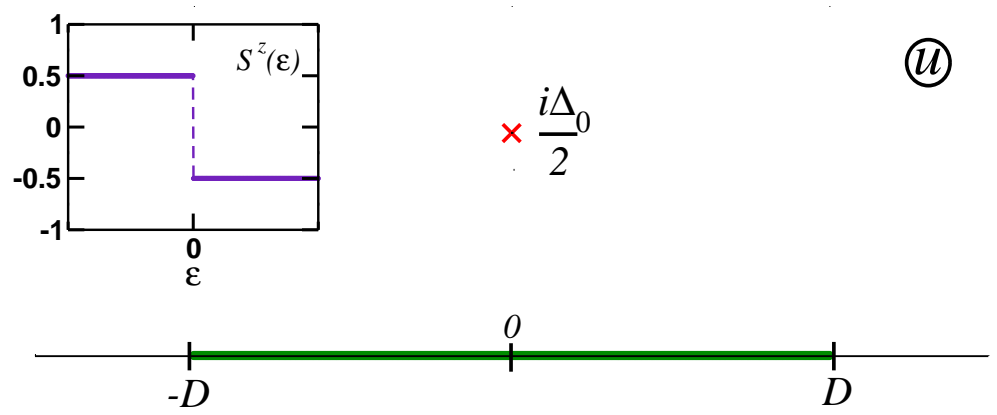

$$
\times \frac{-i \Delta}{2} 0
$$

FIG. 9: (color online) Zeros of $\mathbf{L}^{2}(u)$ for the Fermi ground state in the complex $u$ plane. The single discontinuity $(k=1)$ in the spin component $s^{z}(\epsilon)$ at $\epsilon=0$, see the inset, leads to a single $(2 k-1=1)$ pair of complex double zeros at $\pm i \Delta_{0} / 2$, where $\Delta_{0}$ is the order parameter in the BCS ground state. There is also a line of double real zeros stretching from $-D$ to $D$, where $D$ is the high-energy cutoff on the single-fermion states participating in the BCS Hamiltonian (17). Frequencies $\omega$ of small oscillations around this state are related to the zeros $c$ as $\omega=2 c$, see Sec. III A In the present case, there is a single $(k=1)$ unstable mode that grows with the rate $\gamma=\Delta_{0}$ giving rise to $k=1$ normal-soliton shown in Fig. [1

\section{Fermi ground state}

We saw that in normal stationary states all zeros of $\mathbf{L}^{2}(u)$ are double degenerate and are solutions of the equation $L_{n}(u)=0$, see Eq. (41). The Fermi ground state has all states below the Fermi energy occupied and states above it empty. This corresponds to $2 s_{j}^{z}=l_{j}=-\operatorname{sgn} \epsilon_{j}$. Therefore, the zeros are determined by the following equation:

$$
\sum_{j=1}^{n} \frac{\operatorname{sgn} \epsilon_{j}}{u-\epsilon_{j}}=-\frac{2}{g} .
$$

There are $n$ solutions each one being a double zero of $\mathbf{L}^{2}(u)$. The analysis of Eq. (53) is similar to that of Eq. (45). Eq. (53) has real roots between consecutive $\epsilon_{j}$ except when $\operatorname{sgn} \epsilon_{j}$ changes from 1 
to -1 . Therefore, there is a real root $c_{j}$ in each interval $\left(\epsilon_{j}, \epsilon_{j+1}\right)$ except for the interval containing the Fermi level. Since there are $n-2$ such intervals, $n-2$ roots are real while the remaining two can be complex. Due to the particle-hole symmetry (44) the complex roots must be purely imaginary, see Fig. 9. They also must be complex conjugate to each other as Eq. (53) is invariant under complex conjugation.

In the continuum limit the spacing between $\epsilon_{j}$ vanishes and for the real roots we have $c_{j} \approx \epsilon_{j}$, i.e. $\mathbf{L}^{2}(u)$ has a line of double real zeros stretching from $-D$ to $D$, Fig. 9, To determine the two imaginary roots $\pm i \gamma$, we rewrite Eq. (53) in the integral form

$$
\int_{0}^{D}\left(\frac{d \epsilon}{\epsilon-i \gamma}+\frac{d \epsilon}{\epsilon+i \gamma}\right)=\frac{2}{\lambda}
$$

Using Eq. (26), we obtain in the weak coupling regime $\Delta_{0} \ll D$

$$
\gamma=\frac{\Delta_{0}}{2}
$$

Thus, according to the discussion in the previous subsection, equations of motion (20) linearized around the Fermi ground state show $n-1$ stable modes with oscillation frequencies $\omega_{j} \approx 2 \epsilon_{j}$ and one unstable mode that grows as $e^{2 \gamma t}=e^{\Delta_{0} t}[27,41]$. Note also that the $z$ component of spins $2 s^{z}\left(\epsilon_{j}\right)=-\operatorname{sgn} \epsilon_{j}$ in the Fermi ground state experiences a single jump at the Fermi level.

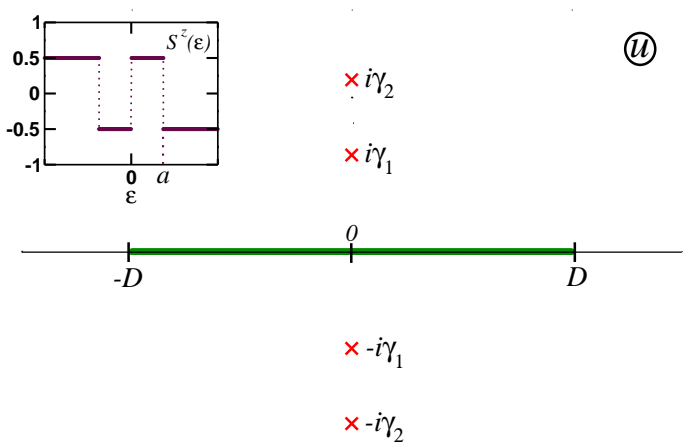

a)

FIG. 10: (color online) Zeros of $\mathbf{L}^{2}(u)$ for an excited normal state characterized by $2 k-1=3$ jumps in the spin component $s^{z}(\epsilon)$ at $\epsilon= \pm a$ (insets). There are $k=2$ pairs of complex conjugate double zeros (identified with crosses) leading to $k=2$ normal-soliton solutions shown in Fig. 2, The complex zeros are determined by $a$ and the ground state gap $\Delta_{0}$, see Eq. (55). a) corresponds to the case $4 a<\Delta_{0}$ and Fig. (2a) and b) corresponds to $4 a>\Delta_{0}$ and Fig. 2b). 


\section{Excited normal state}

Now consider a normal stationary state where $s^{z}\left(\epsilon_{j}\right)$ has three discontinuities. We require $2 s^{z}\left(\epsilon_{j}\right)=-1$ (1) for large positive (negative) $\epsilon_{j}$. Otherwise, the first term in Eq. (22) is not minimized at large $\epsilon_{j}$ and single particle states far from the Fermi level are affected by the pairing interaction, which is unphysical. Under these conditions the total number of discontinuities in $s^{z}\left(\epsilon_{j}\right)$ must be odd. Therefore, the next option after the Fermi ground state that has one jump is a state with three jumps in $s^{z}\left(\epsilon_{j}\right)$.

Let $2 s_{j}^{z}=l_{j}=-\operatorname{sgn} \epsilon_{j}\left(\epsilon_{j}^{2}-a^{2}\right)$, i.e. spins in the interval $\left|\epsilon_{j}\right| \leq a$ point in directions opposite to those in the Fermi ground state, see the insets in Fig. 10. The solutions of $\mathbf{L}^{2}(u)=0$ are determined in the same way as for the Fermi ground state. In the present case, we find that there are $n-4$ double roots located between $\epsilon_{j}$ and $\epsilon_{j+1}$ except when $l_{j}$ and $l_{j+1}$ have different signs. The remaining four double roots can take complex values. In the continuum limit, $\mathbf{L}^{2}(u)$ has a line of double zeros from $-D$ to $D$ and four isolated complex zeros $c=i \gamma_{1,2}$ and $c=-i \gamma_{1,2}$ shown in Fig. 10, where

$$
\gamma_{1,2}=\frac{\Delta_{0}}{4} \pm \sqrt{\frac{\Delta_{0}^{2}}{16}-a^{2}}
$$

Correspondingly, there are two unstable modes (one for each pair of complex conjugate zeros). If $a \leq \Delta_{0} / 4$, Eq. (55) yields real $\gamma_{1,2}$ (Fig. 10a) and the unstable modes grow as $e^{2 \gamma_{1} t}$ and $e^{2 \gamma_{2} t}$. For $a>\Delta_{0} / 4$ we have $c= \pm \mu \pm i \gamma$ (Fig. 10b), where $\mu=\Delta_{0} / 4$ and $\gamma=\sqrt{a^{2}-\Delta_{0}^{2} / 16}$. In this case unstable modes diverge in an oscillatory manner as $e^{ \pm 2 i \mu t} e^{2 \gamma t}$. In general, a normal stationary state with $2 k-1$ discontinuities in $s^{z}\left(\epsilon_{j}\right)$ is characterized by up to $2 k$ complex double zeros of $\mathbf{L}^{2}(u)$ and $k$ unstable modes.

\section{NORMAL SOLITONS}

In this section, we determine solutions of equations of motion (11) that asymptote to normal stationary states at $t \rightarrow \pm \infty$. In particular, we derive equations (44) - (12) for normal solitons, see also Figs. 1, 2, and 3. That these solutions are solitons can be seen in a number of ways. First, these are trajectories that connect an unstable equilibrium to itself, i.e. they start in an unstable stationary state at $t \rightarrow-\infty$ and return to it at $t \rightarrow \infty$. This is typical of solitons [42], see the paragraph preceding Eq. (4). Second, as we will show, in a certain regime the solution splits into a sum of single solitons as it should [43]. Finally, in contrast to the general solution these solutions are in terms of elementary functions [44]. 


\section{A. General $k$-normal-soliton solution}

Consider a general normal stationary state with $2 k-1$ discontinuities in $s^{z}\left(\epsilon_{j}\right)$. Suppose $\mathbf{L}^{2}(u)=L_{n}^{2}(u)$ has $2 k$ complex double zeros $c_{1}, c_{2}, \ldots, c_{2 k}$, i.e. there are $k$ unstable modes in the linear analysis. Let us solve equations of motion for separation variables (36) for this state.

First, we derive a useful equation for the time-dependent gap function $\Delta(t) . L_{n}(u)$ has $2 k$ complex conjugate zeros $c_{1}, c_{2}, \ldots, c_{2 k}$ and $n-2 k$ real zeros. Bringing Eq. (41) to a common denominator, we obtain

$$
L_{n}(u)=-\frac{1}{g} \frac{P_{2 k}(u) R_{n-2 k}(u)}{\prod_{m}\left(u-\epsilon_{m}\right)}
$$

where $P_{2 k}(u)=\prod_{r=1}^{2 k}\left(u-c_{r}\right)$ and $R_{n-2 k}(u)$ represents the contribution of the real zeros. Both these polynomials have real coefficients. Eq. (31) yields

$$
\left[L_{z}(u)-L_{n}(u)\right]\left[L_{z}(u)+L_{n}(u)\right]=-L_{-}(u) L_{+}(u)
$$

This equation implies

$$
\begin{aligned}
L_{z}(u)+L_{n}(u) & =-\frac{2}{g} \frac{S_{k}(u) S_{k}^{*}(u) R_{n-2 k}(u)}{\prod_{m}\left(u-\epsilon_{m}\right)}, \\
L_{z}(u)-L_{n}(u) & =-\frac{g}{2} J_{-} J_{+} \frac{T_{k-1}(u) T_{k-1}^{*}(u) R_{n-2 k}(u)}{\prod_{m}\left(u-\epsilon_{m}\right)}, \\
L_{-}(u) & =J_{-} \frac{S_{k}(u) T_{k-1}(u) R_{n-2 k}(u)}{\prod_{m}\left(u-\epsilon_{m}\right)},
\end{aligned}
$$

where $S_{k}(u)$ and $T_{k-1}(u)$ are polynomials in $u$ of orders $k$ and $k-1$, respectively. The coefficient at highest power of $u$ is equal to unity in all polynomials. The coefficients of $S_{k}^{*}(u)$ are complex conjugate to those of $S_{k}(u)$ and similarly for $T_{k-1}^{*}(u)$. The prefactors in Eqs. (58) and (59) are obtained from large $u$ behavior. For example, Eqs. (28) and (41) imply $L_{z}(u)+L_{n}(u) \approx-2 / g$ at $u \rightarrow \infty$. The right hand side of the first equation in (58) has the same large $u$ asymptote. The polynomial $R_{n-2 k}(u)$ is common to all components of $\mathbf{L}(u)$ since any real zero of $\mathbf{L}^{2}(u)$ is also a zero of $L_{x, y, z}[50]$. Subtracting the second equation in (58) from the first one and using Eq. (56), we derive

$$
P_{2 k}(u)=S_{k}(u) S_{k}^{*}(u)+\frac{|\Delta|^{2}}{4} T_{k-1}(u) T_{k-1}^{*}(u),
$$

where we used $\Delta(t)=g J_{-}(t)$. We will need this equation below to determine $|\Delta(t)|$. 
Now consider equations of motion (36). It follows from Eq. (311) and the definition (32) of separation variables $u_{j}$ that $\mathbf{L}^{2}\left(u_{j}\right)=L_{z}^{2}\left(u_{j}\right)$. Comparing the large $u$ behavior of $\sqrt{\mathbf{L}^{2}(u)} \rightarrow 1 / g$ and $L_{z}(u) \rightarrow-1 / g$, we conclude that $\sqrt{\mathbf{L}^{2}\left(u_{j}\right)}=-L_{z}\left(u_{j}\right)$. Eq. (36) takes the following form:

$$
\begin{aligned}
& \sum_{j=1}^{2 k-1} \frac{u_{j}^{l} d u_{j}}{\widetilde{L}_{z}\left(u_{j}\right) \prod_{m}\left(u_{j}-\epsilon_{m}\right)}=0, \quad l=0, \ldots, 2 k-3, \\
& \sum_{j=1}^{2 k-1} \frac{u_{j}^{2 k-2} d u_{j}}{\widetilde{L}_{z}\left(u_{j}\right) \prod_{m}\left(u_{j}-\epsilon_{m}\right)}=-2 i g d t,
\end{aligned}
$$

where $\widetilde{L}_{z}(u)=L_{z}(u) / R_{n-2 k}$. Eqs. (32) and (59) imply that unfrozen separation variables $u_{1}, \ldots, u_{2 k-1}$ are the roots of $S_{k}(u) T_{k-1}(u)$. Let $u_{1}, \ldots, u_{k-1}$ be the roots of $T_{k-1}(u)$ and $u_{k}, \ldots, u_{2 k-1}$ the roots of $S_{k}(u)$. Eq. (158) reads $\widetilde{L}_{z}\left(u_{j}\right)=\widetilde{L}_{n}\left(u_{j}\right)$ for $j=1, \ldots, k-1$ and $\widetilde{L}_{z}\left(u_{j}\right)=-\widetilde{L}_{n}\left(u_{j}\right)$ for $j=k, \ldots, 2 k-1$, where $\widetilde{L}_{n}(u)=L_{n}(u) / R_{n-2 k}$. Further, using Eq. (56), we obtain from Eq. (61)

$$
\sum_{j=k}^{2 k-1} \frac{u_{j}^{l} d u_{j}}{P_{2 k}\left(u_{j}\right)}-\sum_{j=1}^{k-1} \frac{u_{j}^{l} d u_{j}}{P_{2 k}\left(u_{j}\right)}=-2 i d t \delta_{l, 2 k-2}, \quad l=0, \ldots, 2 k-2 .
$$

Eq. (62) does not contain square roots in contrast to Eq. (36) and can be integrated in elementary functions. To do so, we expand the ratios $u^{l} / P_{2 k}(u)$ in elementary fractions

$$
\frac{u^{l}}{P_{2 k}(u)}=\sum_{m=1}^{2 k} \frac{c_{m}^{l}}{\left(u-c_{m}\right) \prod_{j \neq m}\left(c_{m}-c_{j}\right)}, \quad l<2 k .
$$

This identity can be verified by comparing residues at poles $u=c_{m}$ on both sides. Using expansion (63) in Eq. (62), we obtain

$$
\sum_{m=1}^{2 k} \frac{c_{m}^{l} d x_{m}}{\prod_{j \neq m}\left(c_{m}-c_{j}\right)}=-2 i d t \delta_{l, 2 k-2}
$$

where $l=0, \ldots, 2 k-2$ and

$$
d x_{m}=\sum_{j=k}^{2 k-1} \frac{d u_{j}}{u_{j}-c_{m}}-\sum_{j=1}^{k-1} \frac{d u_{j}}{u_{j}-c_{m}}=d \ln \frac{S_{k}\left(c_{m}\right)}{T_{k-1}\left(c_{m}\right)}, \quad x_{m} \equiv \frac{S_{k}\left(c_{m}\right)}{T_{k-1}\left(c_{m}\right)} .
$$

Integration of Eq. (64) results in

$$
\sum_{m=1}^{2 k} \frac{c_{m}^{l} x_{m}}{\prod_{j \neq m}\left(c_{m}-c_{j}\right)}=-2 i t \delta_{l, 2 k-2}+E\left(c_{l}\right), \quad l=0, \ldots, 2 k-2,
$$

where $E\left(c_{l}\right)$ are the integration constants. These equations are linear in $x_{m}$ with the general solution

$$
x_{m}=-2 i c_{m} t+\widetilde{E}\left(c_{m}\right)+G(t) .
$$


$\widetilde{E}\left(c_{m}\right)$ are new time-independent constants and $G(t)$ is an arbitrary function of $t$.

Using the definition of $x_{m}$ in Eq. (65), we find

$$
\frac{S_{k}\left(c_{m}\right)}{T_{k-1}\left(c_{m}\right)}=-A\left(c_{m}\right) F(t) e^{-2 i c_{m} t}, \quad m=1, \ldots, 2 k,
$$

where $A\left(c_{m}\right)$ are complex constants and $F(t)$ is a function of time to be determined below. Eqs. (68) are $2 k$ linear equations for $2 k-1$ coefficients of polynomials $S_{k}(u)$ and $T_{k-1}(u)$. The compatibility condition for this linear system yields a linear equation for the function $F(t)$. We derive

$$
F(t)=(-1)^{k} 2^{2 k-2} \frac{D_{k-1}}{D_{k}}
$$

where the determinant $D_{r}$ is given by

$$
D_{r}=\left|\begin{array}{lll}
f & \ldots & f^{(r-1)} \\
\vdots & & \vdots \\
f^{(r-1)} & \ldots & f^{2(r-1)}
\end{array}\right|,
$$

$f^{(j)}$ is the $j$ th derivative of the function $f(t)$ with respect to $t$, and

$$
f(t)=\sum_{m=1}^{2 k} \frac{A\left(c_{m}\right) e^{-2 i c_{m} t}}{\prod_{l \neq m}\left(c_{m}-c_{l}\right)} .
$$

To relate $|F(t)|$ to $|\Delta(t)|$, we use Eq. (60). This equation also imposes certain restrictions on complex constants $A\left(c_{m}\right)$. Setting $u=c_{m}$ in Eq. (60) and using the fact that $c_{m}$ are the roots of $P_{2 k}(u), P_{2 k}\left(c_{m}\right)=0$, we obtain

$$
\frac{S\left(c_{m}\right)}{T\left(c_{m}\right)} \frac{S^{*}\left(c_{m}\right)}{T^{*}\left(c_{m}\right)}=-\frac{|\Delta|^{2}}{4}
$$

Note that while the coefficients of the polynomial $S_{m}^{*}(u)$ are complex conjugate to those of $S_{m}(u)$, $S^{*}\left(c_{m}\right)$ is not complex conjugate to $S\left(c_{m}\right)$ since $c_{m}$ is complex. Instead, we have $S^{*}\left(c_{m}\right)=\left[S\left(c_{m}^{*}\right)\right]^{*}$, i.e. $S^{*}\left(c_{m}\right)$ is conjugate to $S\left(c_{m}^{*}\right)$. Using this and Eq. (68), we obtain from Eq. (72)

$$
A\left(c_{m}\right) A^{*}\left(c_{m}^{*}\right)|F(t)|^{2}=-\frac{|\Delta(t)|^{2}}{4},
$$

where $A^{*}\left(c_{m}^{*}\right)$ is the complex conjugate of $A\left(c_{m}^{*}\right)$ - the constant corresponding to the zero $c_{m}^{*}$ complex conjugate to $c_{m}$ (recall that the zeros $c_{j}$ of $\mathbf{L}^{2}(u)$ come in complex conjugate pairs). Eq. (73) implies that the product $A\left(c_{m}\right) A^{*}\left(c_{m}^{*}\right)$ is independent of $m$. With no loss of generality we set

$$
A\left(c_{m}\right) A^{*}\left(c_{m}^{*}\right)=-1
$$


Any other real value will rescale $|F(t)|$ without affecting $|\Delta(t)|$. Therefore, we have $|\Delta(t)|=2|F(t)|$ and

$$
|\Delta(t)|=2^{2 k-1}\left|\frac{D_{k-1}}{D_{k}}\right|
$$

It follows from Eq. (174) that the constants $A\left(c_{m}\right)$ can be parameterized as follows

$$
A\left(c_{l}\right)=e^{\alpha_{l}+i \phi_{l}}, \quad A\left(c_{k+l}\right)=-e^{-\alpha_{l}+i \phi_{l}},
$$

where $\alpha_{l}$ and $\phi_{l}$ are arbitrary real parameters and we ordered the $2 k$ zeros $c_{m}$ so that $c_{k+l}=c_{l}^{*}$ and $\operatorname{Im}\left(c_{l}\right)>0$ for $l=1, \ldots, k$.

Eqs. (75), (70), (71), (68) and (76) fully describe the general $k$-normal-soliton solution (examples for $k=1,2$, and 3 are shown in Figs. 1, 2, and 3, respectively). They contain $2 k$ zeros $c_{m}$ fixed by the normal stationary state corresponding to this solution. This state has $2 k-1$ discontinuities in $s^{z}\left(\epsilon_{m}\right)$. The zeros $c_{m}$ are the roots of the equation $L_{n}(u)=0$, where $L_{n}(u)$ is given by Eq. (41). The $2 k$ real parameters $\alpha_{l}$ and $\phi_{l}$ in Eq. (76) are arbitrary. That the general $k$-normal-soliton should be indeed characterized by $2 k$ arbitrary real parameters is seen from the discussion in the paragraph following Eq. (35). As mentioned there (see Refs. 29 and 33 for details), a real double zero of $\mathbf{L}^{2}(u)$ effectively reduces the number of degrees of freedom (spins) by one. Since in the present case we have $n-2 k$ such roots, it can be described by $2 k$ effective spins. Then, there are $4 k$ initial conditions (two angles per each spin). $2 k$ of these are determined by the $2 k$ integrals of motion $c_{m}$, while the other $2 k$ correspond to $\alpha_{l}$ and $\phi_{l}$.

\section{B. Matching soliton constants to spin configuration at large negative time}

Here we show that the $k$-soliton (70) tends to a normal stationary state in $t \rightarrow \pm \infty$ limits and relate the constants $\alpha_{l}$ and $\phi_{l}$ to the deviations of spins from this state at large negative times.

First, let us evaluate expression (70) for large negative $t$. To this end, we keep in Eq. (71) only the exponents that diverge in the $t \rightarrow-\infty$ limit, i.e. the $k$ terms that have $\operatorname{Im}\left(c_{m}\right)<0$. After some manipulations with the rows of determinants $D_{k}$ and $D_{k-1}$, we derive

$$
|\Delta(t)|=2\left|\sum_{m=1}^{k} \frac{e^{-2 i c_{m} t} e^{\alpha_{m}+i \phi_{m}}\left(c_{m}-c_{m}^{*}\right) \prod_{i}\left(c_{m}-c_{i}^{*}\right)}{\prod_{i \neq m}\left(c_{m}-c_{i}\right)}\right| .
$$

We see that $|\Delta(t)| \propto e^{-2 \gamma t}$ at large negative $t$, where $\gamma$ is the minimum of $\left|\operatorname{Im}\left(c_{m}\right)\right|$. Quantities $J_{ \pm}(t)$ and $F(t)$ behave in the same way as they are proportional to $|\Delta(t)|$. According to Eq. (68) as $t \rightarrow-\infty$ either $S_{k}\left(c_{m}\right) \rightarrow 0$ or $T_{k-1}\left(c_{m}\right) \rightarrow 0$ except for the zero $c_{m}$ with $\operatorname{Im}\left(c_{m}\right)=i \gamma$. Since the 
unfrozen separation variables are the roots of either $S_{k}(u)$ or $T_{k-1}(u)$ (see Eqs. (32) and (59)), they must tend to their stationary state positions $c_{m}$. Observe also that since $J_{ \pm}(t) \rightarrow 0$, the second equation in (57) and Eq. (59) mean $L_{z}(u) \rightarrow L_{n}(u)$ and $L_{-}(u) \rightarrow 0$. It follows from the definition (28) of $\mathbf{L}(u)$ and Eq. (41) that $s_{j}^{x, y} \rightarrow 0$ and $s_{j}^{z} \rightarrow l_{j} / 2$. Thus, the $k$-normal-soliton tends to the normal stationary state that has the same values of zeros $c_{i}$. The analysis of the $t \rightarrow \infty$ limit is completely analogous.

Next, consider the limiting stationary state. There are $2 k$ zeros $c_{i}$ and only $2 k-1$ unfrozen separation variables, i.e. one of the zeros $c_{i}$ (say $c_{r}$ ) remains vacant. Suppose spins deviate from this stationary state keeping the values of integrals of motion $c_{i}$ the same. Since $J_{-}=0$ in normal states, Eq. (33) yields to the linear order in the deviation

$$
s_{j}^{-}=J_{-} \frac{R_{n-2 k}\left(\epsilon_{j}\right) \prod_{i}\left(\epsilon_{j}-u_{i}^{(0)}\right)}{\prod_{i \neq j}\left(\epsilon_{j}-\epsilon_{i}\right)},
$$

where $u_{i}^{(0)}=c_{i}$ are the stationary positions of the unfrozen separation variables and $R_{n-2 k}\left(\epsilon_{j}\right)$ is the contribution of the frozen ones. The frozen variables are located in real zeros of $L_{n}(u)$, which are also the zeros of $R_{n-2 k}(u)$, see Eq. (56). Further, Eqs. (41) and (56) imply

$$
-\frac{1}{g}+\sum_{j=1}^{n} \frac{l_{j}}{2\left(u-\epsilon_{j}\right)}=-\frac{1}{g} \frac{R_{n-2 k}(u)\left(u-c_{r}\right) \prod_{i}\left(u-u_{i}^{(0)}\right)}{\prod_{m}\left(u-\epsilon_{m}\right)} .
$$

Equating the residues at poles $u=\epsilon_{j}$ on both sides, we obtain

$$
\frac{R_{n-2 k}\left(\epsilon_{j}\right) \prod_{i}\left(\epsilon_{j}-u_{i}^{(0)}\right)}{\prod_{i \neq j}\left(\epsilon_{j}-\epsilon_{i}\right)}=-\frac{l_{j} g}{2\left(\epsilon_{j}-c_{r}\right)} .
$$

Substituting this into Eq. (78), we find

$$
s_{j}^{-}(t)=-g J_{-}(t) \frac{l_{j}}{2\left(\epsilon_{j}-c_{r}\right)} .
$$

Finally, $J_{-}(t)$ is determined from Eq. (42), which was also derived in a linear analysis around normal stationary states. The difference is that there we considered generic deviations when the integrals of motion $c_{i}$ also deviate from their stationary state values. Nevertheless, Eq. (42) is the same in both cases and integrating it, we obtain

$$
\begin{aligned}
& \Delta(t)=g J_{-}(t)=\beta_{r} e^{-2 i c_{r} t}, \\
& s_{j}^{-}(t)=-\beta_{r} \frac{l_{j} e^{-2 i c_{r} t}}{2\left(\epsilon_{j}-c_{r}\right)} .
\end{aligned}
$$

These are particular solutions of the linearized equations of motion. They describe an unstable mode with complex "frequency" $2 c_{r}$. 
The general solution (with $c_{i}$ fixed to their stationary state values) is a superposition of all modes, i.e.

$$
\begin{aligned}
& \Delta(t)=g J_{-}(t)=\sum_{r=1}^{k} \beta_{r} e^{-2 i c_{r} t}, \\
& s_{j}^{-}(t)=-\sum_{r=1}^{k} \beta_{r} \frac{l_{j} e^{-2 i c_{r} t}}{2\left(\epsilon_{j}-c_{r}\right)} .
\end{aligned}
$$

Note that these equations contain only $c_{r}$ such that $\operatorname{Im}\left(c_{r}\right)<0$, same as in Eq. (77), to insure that the deviations are indeed small at large negative $t$. Comparing Eqs. (83) and (77), we find

$$
\beta_{m}=\frac{2\left(c_{m}-c_{m}^{*}\right) \prod_{i}\left(c_{m}-c_{i}^{*}\right)}{\prod_{i \neq m}\left(c_{m}-c_{i}\right)} e^{\alpha_{m}+i \phi_{m}}, \quad \operatorname{Im}\left(c_{m}\right)<0 .
$$

Eqs. (85) and (84) specify deviations of spins from their normal stationary state positions necessary to generate the $k$-normal-soliton (75). Indeed, an arbitrary choice of real $\alpha_{m}, \phi_{m}$, and large negative $t=t_{0}$ determines $\beta_{m}$ and deviations of spins (84). Equations of motion (20) started at $t=t_{0}$ with these initial conditions produce the $k$-soliton solution (75) with the same values of $c_{i}$ as those in the stationary state. On the other hand, note that generic deviations of spins will modify $c_{i}$, see e.g. the text following Eq. (39), and will not lead to solitons.

\section{Examples of 1 and 2-normal-solitons}

In this subsection, we consider $k=1$ and $k=2$ normal solitons in more detail, see also the Introduction.

1-normal-soliton. The single normal soliton solution (Fig. 1) has been previously found in Ref. 27. Here we derive it from the general $k$-soliton (75) as its simplest particular case to illustrate our construction of multi-soliton solutions. In this case $k=1$ and $\mathbf{L}^{2}(u)$ has two complex double zeros $c_{1}=c_{2}^{*} \equiv \mu+i \gamma$ as illustrated in Fig. 9. The corresponding normal stationary state has a single discontinuity in the $z$ component of spin (inset in Fig. 9), i.e. it is the Fermi ground state, see Sec. IIIB, We have seen that in the particle-hole symmetric case $2 s_{j}^{z}=-\operatorname{sgn} \epsilon_{j}, \mu=0$, and $\gamma=\Delta_{0} / 2$.

Eqs. (73), (71), and (69) yield

$$
A\left(c_{1}\right)=e^{\alpha+i \phi}, \quad A\left(c_{2}\right)=-e^{-\alpha+i \phi}, \quad F=-i \frac{\gamma}{\cosh (2 \gamma t+\alpha)} e^{2 i \mu t-i \phi} .
$$

and

$$
|\Delta(t)|=2|F(t)|=\frac{2 \gamma}{\cosh (2 \gamma t+\alpha)}
$$


Graphically, the single soliton is represented by a single peak located at $t_{0}=-\alpha / 2 \gamma$, see Fig. 1. The parameter $\gamma$ controls the width and the height of the peak.

There is $2 k-1=1$ unfrozen separation variable $u_{1}$. Therefore, $S_{k}(u)=u-u_{1}$ and $T_{k-1}(u)=1$. Eq. (68) implies

$$
u_{1}(t)=\mu-i \gamma \tanh (2 \gamma t+\alpha)
$$

Note that $u_{1} \rightarrow \mu \pm i \gamma=c_{1,2}$ as $t \rightarrow \mp \infty$ in agreement with the results of the previous subsection. The separation variable starts from the complex zero $\mu+i \gamma$ of $\mathbf{L}^{2}(u)$ at $t=-\infty$ and goes to the complex conjugate zero $\mu-i \gamma$ at $t=\infty$ along the straight line connecting the two zeros shown in Fig. 9.

Individual spin components can be derived from Eqs. (56), (59), and (87). We have

$$
L_{n}(u)=-\frac{1}{g} \frac{\left(u-c_{1}\right)\left(u-c_{1}^{*}\right) R_{n-2}(u)}{\prod_{m}\left(u-\epsilon_{m}\right)}, \quad L_{-}(u)=J_{-} \frac{\left(u-u_{1}\right) R_{n-2}(u)}{\prod_{m}\left(u-\epsilon_{m}\right)} .
$$

Therefore,

$$
L_{-}(u)=-\Delta(t) \frac{\left(u-u_{1}\right)}{\left(u-c_{1}\right)\left(u-c_{1}^{*}\right)} L_{n}(u)
$$

Using expression (41) with $l_{j}=-\operatorname{sgn} \epsilon_{j}$ and comparing the residues at poles at $u=\epsilon_{j}$ on both sides of the above equation, we obtain[27]

$$
s_{j}^{-}(t)=s_{j}^{x}(t)+i s_{j}^{y}(t)=\Delta(t) \frac{\left[\epsilon_{j}-u_{1}(t)\right] \operatorname{sgn} \epsilon_{j}}{2\left[\left(\epsilon_{j}-\mu\right)^{2}+\gamma^{2}\right]} .
$$

Similarly, the second equation in Eq. (58) yields

$$
s_{j}^{z}(t)=\frac{\operatorname{sgn} \epsilon_{j}}{2}\left[\frac{|\Delta(t)|^{2}}{\left(\epsilon_{j}-\mu\right)^{2}+\gamma^{2}}-1\right] .
$$

2-normal-soliton. Now $k=2$ and $\mathbf{L}^{2}(u)$ has four complex zeros, Fig. 10, The limiting excited normal state exhibits $2 k-1=3$ jumps in $s^{z}\left(\epsilon_{j}\right)$, see the inset in Fig. 10. In Sec. IIIB, we considered such a stationary state with $2 s_{j}^{z}=-\operatorname{sgn} \epsilon_{j}\left(\epsilon_{j}^{2}-a^{2}\right)$ and determined the corresponding complex zeros.

For $a \leq \Delta_{0} / 4$ these zeros are purely imaginary (Fig. 10a), $c_{1}=i \gamma_{1}, c_{2}=i \gamma_{2}, c_{3}=-i \gamma_{1}$, and $c_{4}=-i \gamma_{2}$, where $\gamma_{1,2}$ are given by Eq. (55). Eq. (75) yields

$$
|\Delta(t)|=A\left|\frac{h(t)}{h(t) \ddot{h}(t)-\dot{h}^{2}(t)}\right|
$$

where $A=4\left|\gamma_{2}^{2}-\gamma_{1}^{2}\right|$ and

$$
h(t)=e^{i \phi_{1}} \frac{\cosh \left(2 \gamma_{1} t+\alpha_{1}\right)}{2 \gamma_{1}}+e^{i \phi_{2}} \frac{\cosh \left(2 \gamma_{2} t+\alpha_{2}\right)}{2 \gamma_{2}} .
$$


The plot of the 2-normal-soliton (88) displays two peaks, see Fig. 2. Parameters $\alpha_{1,2}$ determine the location of the peaks in time, while $\gamma_{1,2}$ control their widths and heights. The 2 -soliton can be viewed as a nonlinear superposition of two single solitons. At large separation between solitons in time, $\left|\alpha_{1}-\alpha_{2}\right| \gg 1$, we obtain from Eq. (88)

$$
|\Delta(t)| \approx \frac{2 \gamma_{1}}{\cosh \left(2 \gamma_{1} t+\alpha_{1}+\eta\right)}+\frac{2 \gamma_{2}}{\cosh \left(2 \gamma_{2} t+\alpha_{2}-\eta\right)},
$$

where the phase shift $\eta$ is

$$
\tanh \eta=\operatorname{sgn}\left(\alpha_{2}-\alpha_{1}\right) \frac{2 \gamma_{1} \gamma_{2}}{\gamma_{1}^{2}+\gamma_{2}^{2}}
$$

In deriving Eq. (90) we neglected the terms of relative smallness $e^{-\left|\alpha_{1}-\alpha_{2}\right|}$. We see that at large separation, the 2-normal-soliton reduces to a simple sum of two single solitons as shown in Fig. 2 , This is a general property of solitons and one can show that the general $k$-normal-soliton (75) also obeys this rule, see e.g. Fig. 3 and Eq. (12). For small separation the two peaks merge into one.

When $a>\Delta_{0} / 4$ in Eq. (55) the four roots of $\mathbf{L}^{2}(u)$ have the form $\pm \mu \pm i \gamma$ (Fig. 10 $\mathrm{b}$ ), where $\mu=\Delta_{0} / 4$ and $\gamma=\sqrt{a^{2}-\Delta_{0}^{2} / 16}$. In this case the 2-normal soliton is again given by Eq. (88) where now $A=16 \mu \sqrt{\mu^{2}+\gamma^{2}}$ and

$$
h(t)=e^{-2 i \mu t+i \phi_{1}} \frac{\cosh \left(2 \gamma t+\alpha_{1}-i \beta\right)}{2 \gamma}+e^{2 i \mu t+i \phi_{2}} \frac{\cosh \left(2 \gamma t+\alpha_{2}+i \beta\right)}{2 \gamma} .
$$

An additional feature as compared to Eq. (89) is that here the two terms "rotate" with frequency $4 \mu$ with respect to one another. For large separation, $\left|\alpha_{1}-\alpha_{2}\right| \gg 1$, this has no effect - the plot of $|\Delta(t)|$ still shows two peaks well separated in time, dashed lines in Fig. $2 \mathrm{~b}$. Now the peaks are the same, i.e. $\gamma_{1}=\gamma_{2}=\gamma$ in Eq. (89). In contrast, when the separation is small there is a single peak as in the 2-soliton (89) but with an amplitude modulated by an oscillation with frequency $\omega \sim 4 \mu=\Delta_{0}$, see Fig $2 \mathrm{~b}$.

\section{ANOMALOUS SOLITONS}

In this section, we construct 1- and 2-anomalous-solitons (see also Figs. 4 and 5) - solutions of Bogoliubov-de Gennes equations for $|\Delta(t)|$ that asymptote to anomalous stationary states (23) as $t \rightarrow \pm \infty$. These solutions show the same solitonic signatures as normal solitons, see the introductory paragraph in Sec. IV] In particular, they are expressed in terms of exponentials and multi-solitons break up into a sum of well separated single anomalous solitons in a certain limit. 


\section{A. Single anomalous soliton as a special case of a 3 -spin solution}

A single soliton corresponds to the anomalous state with one unstable mode in the linear analysis, i.e. $\mathbf{L}^{2}(u)$ has two double complex zeros in addition to single zeros $u= \pm i \Delta_{a}$, see Sec. IIIA and Fig. 7. We considered a state of this type in Sec. IIIB In this example, spins in the energy interval $(-a, a)$ are flipped; $e_{m}=\operatorname{sgn}\left(\left|\epsilon_{m}\right|-a\right)$ in Eq. (23) as shown in Fig. 7 (inset). In other words, Cooper pairs for single particle states $-a \leq \epsilon \leq a$ are excited. This state is particlehole symmetric (44) and the complex zeros of $\mathbf{L}^{2}(u)$ are therefore purely imaginary, $u= \pm i \gamma$. As we have shown in Sec. IIIA, this anomalous state is unstable for $\gamma>\Delta_{a}$.

For the particle-hole symmetric case equations of motion (20) have the following form:

$$
\dot{s}_{j}^{x}=-2 \epsilon_{j} s_{j}^{y}, \quad \dot{s}_{j}^{z}=-2 \Delta s_{j}^{y}, \quad \dot{s}_{j}^{y}=2 \Delta s_{j}^{z}+2 \epsilon_{j} s_{j}^{x},
$$

where $\Delta=g \sum_{j} s_{j}^{x}$ is real since $\sum_{j} s_{j}^{y}=0$ at all times. Let us solve Eq. (92) under the condition that at $t \rightarrow-\infty$ the solution asymptotes to the above anomalous state. As mentioned below Eq. (32) and detailed in Refs. 29 and 33 , when $\mathbf{L}^{2}(u)$ has $m$ complex conjugate zeros (the remaining $2 n-2 m$ zeros are real) the problem is reduced to solving equations of motion (92) for $m$ effective spins. In the present case $m=3$ (counting the pair of double zeros as two pairs) and therefore we will need to solve Eq. (92) for three spins.

This reduction can be seen in Eqs. (34) and (35). Suppose $Q_{2 n}(u)$ has only three pairs of complex conjugate roots $\left(c_{1}, c_{1}^{*}\right),\left(c_{2}, c_{2}^{*}\right)$, and $\left(c_{3}, c_{3}^{*}\right)$. There are only $3-1=2$ unfrozen separation variables, while the remaining $n-3$ are frozen into the $n-3$ double real roots of $Q_{2 n}(u)$, see the text following Eq. (32). Suppose $c$ is a real root and let $u_{n-1}=c$. Then $Q_{2 n}\left(u_{j}\right)$ contains a factor $\left(u_{j}-c\right)^{2}$ which cancels $u_{j}-u_{n-1}=u_{j}-c$ in the denominator of Eq. (34). This cancellation occurs for all frozen separation variables and we obtain

$$
\begin{aligned}
& \dot{u}_{j}=\frac{2 i \sqrt{Q_{6}\left(u_{j}\right)}}{\prod_{m \neq j}\left(u_{j}-u_{m}\right)}, \quad j, m=1,2, \\
& \dot{J}_{-}=2 i J_{-}\left(u_{1}+u_{2}\right)
\end{aligned}
$$

where $Q_{6}(u)=\prod_{i=1}^{3}\left(u-c_{i}\right)\left(u-c_{i}^{*}\right)$. Eq. (194) follows from Eq. (35), since $\sum_{j} \epsilon_{j}, J_{z}$, and the sum of frozen separation variables, $\sum_{j=3}^{n-1} u_{j}$ vanish due to the particle-hole symmetry [52]. We see that equations of motion (93) and (94) are exactly the same as (34) and (35) for $n=3$ in the particle-hole symmetric case. Since the latter equations and Eq. (92) are equivalent, Eqs. (93) and (94) describe the motion of three effective spins $\mathbf{S}_{1}, \mathbf{S}_{2}$, and $\mathbf{S}_{3}$. Note that $J_{-}(t)$ and consequently $\Delta(t)=g J_{-}(t)$ are the same in both problems. Moreover, one can show [29, 33] that the original 
spins are linearly related to the effective ones, i.e.

$$
\mathbf{s}_{j}=a_{j} \mathbf{S}_{1}+b_{j} \mathbf{S}_{2}+d_{j} \mathbf{S}_{3}
$$

Thus, to construct a single anomalous soliton, we need to solve Eq. (92) for three spins.

First, let us obtain a general 3-spin solution for which $Q_{6}(u)$ has three distinct pairs of complex conjugate roots. As discussed above, the soliton corresponds to the special case when two of these pairs, $\pm i \gamma$, are degenerate. The third pair is $u= \pm i \Delta_{a}$ and therefore $Q_{6}(u)=\left(u^{2}+\gamma^{2}\right)^{2}\left(u^{2}+\Delta_{a}^{2}\right)$. The particle-hole symmetry of the 3 -spin problem implies $\epsilon_{1}=-\epsilon, \epsilon_{2}=0, \epsilon_{3}=\epsilon$ and

$$
S_{1}^{x}=S_{3}^{x} \equiv S_{x}, \quad-S_{1}^{y, z}=S_{3}^{y, z} \equiv S_{y, z}, \quad S_{2}^{x}=-\frac{1}{2}, \quad S_{2}^{y, z}=0 .
$$

Using $\Delta=g \sum_{m=1}^{3} S_{m}^{x}=2 g S_{x}-g / 2$ and integrating Eq. (92), we determine the effective spins

$$
S_{x}=\frac{\Delta}{2 g}+\frac{1}{2}, \quad S_{y}=-\frac{\dot{\Delta}}{4 g \epsilon}, \quad S_{z}=\frac{\Delta^{2}}{4 g \epsilon}+C,
$$

where $C$ is an integration constant. Combining Eqs. (97) and (95), we derive the original spins in terms of $\Delta(t)$,

$$
s_{j}^{x}=A_{j} \Delta+F_{j}, \quad s_{j}^{y}=B_{j} \dot{\Delta}, \quad s_{j}^{z}=C_{j} \Delta^{2}+D_{j},
$$

where $A_{j}, B_{j}, C_{j}, D_{j}$, and $F_{j}$ are time-independent. The constants $B_{j}, C_{j}$, and $D_{j}$ are odd in $\epsilon_{j}$, while $A_{j}$ and $F_{j}$ are even by particle-hole symmetry (44), i.e. $B_{j} \equiv B\left(\epsilon_{j}\right)=-B\left(-\epsilon_{j}\right)$ etc. Since $\Delta=g \sum_{j} s_{j}^{x}$ we also have

$$
g \sum_{j=1}^{n} A_{j}=1, \quad \sum_{j=1}^{n} F_{j}=0 .
$$

Eq. (98) is similar to the ansatz of Ref. 27, which is obtained by setting $F_{j}=0$. Nevertheless, this difference is important as this ansatz yields 2-spin solutions [29], while here we construct 3-spin ones.

Substituting Eq. (98) into equations of motion (92), we find

$$
\begin{aligned}
& A_{j}=-2 \epsilon_{j} B_{j}, \quad C_{j}=-B_{j}, \quad F_{j}=\frac{2 c_{1} B_{j}}{\epsilon_{j}}, \quad D_{j}=2\left(\epsilon_{j}^{2}-c_{2}\right) B_{j} \\
& B_{j}=-\frac{\epsilon_{j} e_{j}}{4 \sqrt{Q_{6}\left(\epsilon_{j}\right)}}, \quad Q_{6}(u)=u^{2}\left(u^{2}-c_{2}\right)^{2}+c_{1}^{2}-c_{3} u^{2},
\end{aligned}
$$

where $e_{j}= \pm 1$. Since $B_{j}$ is odd, $e_{j}$ must be even, $e\left(\epsilon_{j}\right)=e\left(-\epsilon_{j}\right)$. The polynomial $Q_{6}(u)$ is the same spectral polynomial that appears in Eq. (93), see the discussion of few spin solutions in 
Refs. 29 and 33. The coefficients of the polynomial $Q_{6}(u)$ are constrained by Eq. (99). Plugging Eq. (100) into Eq. (99), we obtain

$$
\sum_{j=1}^{n} \frac{e_{j}}{\sqrt{Q_{6}\left(\epsilon_{j}\right)}}=0, \quad \sum_{j=1}^{n} \frac{\epsilon_{j}^{2} e_{j}}{\sqrt{Q_{6}\left(\epsilon_{j}\right)}}=\frac{2}{g},
$$

which provides two constraints on three parameters $c_{1}, c_{2}$, and $c_{3}$. Thus, 3 -spin solutions constructed here are a one parameter family of solutions to Eq. (92).

It remains to determine $\Delta(t)$ for 3 -spin solutions. The equation for $\Delta(t)$ can be obtained from the condition that the length of spins is conserved by the evolution, $\mathbf{s}_{j}^{2}=1 / 4$. With the help of Eqs. (98) and (100) this condition reduces to

$$
\dot{\Delta}^{2}=-P_{4}(\Delta), \quad P_{4}(\Delta)=\Delta^{4}+4 c_{2} \Delta^{2}-8 c_{1} \Delta+4 c_{3} .
$$

For general $P_{4}(\Delta)$ the solution of this equation is an elliptic function. Here we are only interested in an anomalous soliton. As discussed above, it corresponds to a special choice of the spectral polynomial $Q_{6}(u)=\left(u^{2}+\gamma^{2}\right)^{2}\left(u^{2}+\Delta_{a}^{2}\right)$. According to the expression for $Q_{6}(u)$ in Eq. (100), this implies

$$
c_{1}=-\gamma^{2} \Delta_{a}, \quad c_{2}=-\frac{\Delta_{a}^{2}}{2}-\gamma^{2}, \quad c_{3}=\frac{\Delta_{a}^{4}}{4}-\gamma^{2} \Delta_{a}^{2}
$$

For these values of the parameters, Eq. (102) for the order parameter takes the form

$$
\dot{\Delta}^{2}=-\left(\Delta-\Delta_{a}\right)^{2}\left(\Delta^{2}+2 \Delta \Delta_{a}+\Delta_{a}^{2}-4 \gamma^{2}\right) .
$$

Now the fourth order polynomial on the right hand side has a double root $\Delta_{a}$, which means that Eq. (103) can be solved by elementary means. Note also that the stationary state value $\Delta(t)=\Delta_{a}$ is also a solution. In terms of a new variable $y=\left(\Delta-\Delta_{a}\right)^{-1}$ Eq. (103) reads $\dot{y}^{2}=\lambda^{2} y^{2}-4 \Delta_{a} y-1$, where $\lambda=2 \sqrt{\gamma^{2}-\Delta_{a}^{2}}$. We obtain

$$
\Delta(t)-\Delta_{a}=\frac{\lambda^{2}}{2 \Delta_{a} \pm 2 \gamma \cosh (\lambda t+\alpha)}, \quad \lambda=2 \sqrt{\gamma^{2}-\Delta_{a}^{2}} .
$$

The constraints (101) become the gap equation (24) and the equation determining imaginary zeros $\pm i \gamma$ of $\mathbf{L}^{2}(u)$

$$
\sum_{j} \frac{e_{j}}{\left(\epsilon_{j}^{2}+\gamma^{2}\right) \sqrt{\epsilon_{j}^{2}+\Delta_{a}^{2}}}=0, \quad \sum_{j} \frac{e_{j}}{\sqrt{\epsilon_{j}^{2}+\Delta_{a}^{2}}}=\frac{2}{g} .
$$

We solved these equations in Sec. IIIB, see Eqs. (48) and (50). 
Finally, Eq. (98) together with Eqs. (100) and (104) yield the individual spin components for a single anomalous soliton

$$
\begin{aligned}
& s_{j}^{x}(t)=\frac{e_{j} \epsilon_{j}^{2}\left(\Delta(t)-\Delta_{a}\right)}{2\left(\epsilon_{j}^{2}+\gamma^{2}\right) \sqrt{\epsilon_{j}^{2}+\Delta_{a}^{2}}}+\frac{e_{j} \Delta_{a}}{2 \sqrt{\epsilon_{j}^{2}+\Delta_{a}^{2}}}, \\
& s_{j}^{y}(t)=-\frac{e_{j} \epsilon_{j} \dot{\Delta}(t)}{4\left(\epsilon_{j}^{2}+\gamma^{2}\right) \sqrt{\epsilon_{j}^{2}+\Delta_{a}^{2}}}, \\
& s_{j}^{z}(t)=\frac{e_{j} \epsilon_{j}\left(\Delta^{2}(t)-\Delta_{a}^{2}\right)}{4\left(\epsilon_{j}^{2}+\gamma^{2}\right) \sqrt{\epsilon_{j}^{2}+\Delta_{a}^{2}}}-\frac{e_{j} \epsilon_{j}}{2 \sqrt{\epsilon_{j}^{2}+\Delta_{a}^{2}}} .
\end{aligned}
$$

Eqs. (104) and (105) describe a single anomalous soliton solution to the equations of motion (11) and (20). Note that for $t \rightarrow \pm \infty$ the order parameter $\Delta(t) \rightarrow \Delta_{a}$ and the spin components tend to their stationary state values (23). For $\Delta_{a}=0$ the anomalous soliton (104) turns into the normal one (86). Graphically, the anomalous soliton is represented by a single peak similarly to the normal soliton, see Fig. 4. Parameters $\Delta_{a}$ and $\gamma$ control its width and height, while $\alpha$ determines its position in time.

\section{B. 2-anomalous-soliton solutions}

Two and higher anomalous solitons can be derived by solving equations of motion for the separation variables (36) similarly to the construction of normal solitons above. The $k$-anomaloussoliton corresponds to a root diagram of $\mathbf{L}^{2}(u)$ with $k$ double complex zeros $c_{1}, \ldots, c_{k}$ and a pair

of single zeros $\pm i \Delta_{a}$. Then, the denominator of Eq. (36) is $\sqrt{u^{2}+\Delta_{a}^{2}} \prod_{i}\left(u-c_{i}\right)$, i.e. only a second order polynomial remains under the square root. In this case, Eq. (36) can be integrated in elementary functions. However, here we restrict ourselves to the 2-soliton case and adopt a simpler approach to construct it.

Spin components of the anomalous stationary state, to which the $k$-soliton asymptotes at large times, display $2 k$ discontinuities. We analyzed an example with four discontinuities in Sec. IIB see also Fig. 8, In this example spins in energy intervals $(-b,-a)$ and $(a, b)$ are flipped, which means $e_{m}=\operatorname{sgn}\left(\left|\epsilon_{m}\right|-a\right)\left(\left|\epsilon_{m}\right|-b\right)$ in Eq. (23). $\mathbf{L}^{2}(u)$ has four complex double zeros $\pm i \gamma_{1,2}$ in addition to single zeros $\pm i \Delta_{a}$ as illustrated in Fig. 8. We assume $\gamma_{2}>\gamma_{1}>\Delta_{a}$. Therefore, there are two unstable modes in linear analysis with growth rates $\lambda_{1,2}=2\left(\gamma_{1,2}^{2}-\Delta_{a}^{2}\right)^{1 / 2}$, see Sec. IIIA. The 2-anomalous soliton must have the following properties: a) $\Delta(t) \rightarrow \Delta_{a}$ as $t \rightarrow \pm \infty$ while 
at large $t$ it should reproduce the linear analysis, b) for $\Delta_{a}=0$ it should be equivalent to the 2-normal-soliton described by Eqs. (88) and (89), and c) in a certain regime the 2-soliton must break up into a sum of two single solitons (104). This suggests the following ansatz for the 2-soliton

$$
\begin{aligned}
& \Delta(t)-\Delta_{a}=\frac{f}{f \ddot{f}-\dot{f}^{2}}, \\
& f=a_{0}+\frac{a_{1}}{\lambda_{1}} \cosh \left(\lambda_{1} t+\alpha_{1}\right)+\frac{a_{2}}{\lambda_{2}} \cosh \left(\lambda_{2} t+\alpha_{2}\right),
\end{aligned}
$$

where $a_{0}, a_{1}$, and $a_{2}$ are time-independent parameters. To determine them, we require that for $\left|\alpha_{2}-\alpha_{1}\right| \gg 1$ the 2-soliton be well approximated by a sum of two single anomalous solitons (cf. Eq. (901) )

$$
\Delta(t)-\Delta_{a} \approx \frac{\lambda_{1}^{2}}{2 \Delta_{a} \pm 2 \gamma_{1} \cosh \left(\lambda_{1} t+\alpha_{1}+\eta\right)}+\frac{\lambda_{2}^{2}}{2 \Delta_{a} \pm 2 \gamma_{2} \cosh \left(\lambda_{2} t+\alpha_{2}-\eta\right)}
$$

Neglecting terms of relative smallness $e^{-\left|\alpha_{1}-\alpha_{2}\right|}$ in Eq. (106), we indeed obtain Eq. (108) when $\tanh (\eta / 2)=\lambda_{1} / \lambda_{2}$ and

$$
f=\frac{2 \Delta_{a}}{\lambda_{1}^{2} \lambda_{2}^{2}} \pm \frac{2 \gamma_{1}}{\lambda_{1}^{2}\left(\lambda_{2}^{2}-\lambda_{1}^{2}\right)} \cosh \left(\lambda_{1} t+\alpha_{1}\right) \pm \frac{2 \gamma_{2}}{\lambda_{2}^{2}\left(\lambda_{2}^{2}-\lambda_{2}^{2}\right)} \cosh \left(\lambda_{2} t+\alpha_{2}\right), \quad \lambda_{1,2}=2 \sqrt{\gamma_{1,2}^{2}-\Delta_{a}^{2}}
$$

Further, one can verify that the 2-anomalous-soliton given by Eqs. (106) and (109) also has the properties a) and b) discussed above. Its plot consists of two peaks levelling off to the stationary value $\Delta_{a}$ at large times, see Fig. 5. The amplitudes and the widths of these peaks are determined by parameters $\gamma_{1}, \gamma_{2}$, and $\Delta_{a}$.

\section{CONCLUSION}

In this paper, we constructed soliton solutions of time-dependent Bogoliubov-de Gennes equations (11) or, equivalently, Gorkov equations (20) describing the collisionless dynamics of a fermionic superfluid. There are two types of solitons. Normal solitons asymptote at $t \rightarrow \pm \infty$ to normal stationary states (27), which are simultaneous eigenstates of the mean-field BCS Hamiltonian (3) and the Fermi gas. These states are characterized by zero order parameter, $\Delta=0$. We have derived the general $k$-normal-soliton solution, Eqs. (70), (71), and (75), and matched the soliton constants to small deviations from the corresponding stationary state. We considered the 2-soliton example (88) in detail and related its parameters to those of the asymptotic stationary state. Examples of $k=1,2$, and 3 normal soliton solutions are shown in Fig. 1, 2, and 3, At large separation between the solitons, the $k$-soliton becomes a simple sum of $k$ single solitons, see e.g. Eq. (90) and Figs. 2 and 3 , 
Anomalous solitons asymptote to unstable eigenstates of the mean-field BCS Hamiltonian (23) with nonzero anomalous average. We have obtained one, Eqs. (104), (105), and Fig. 4, and two, Eqs. (106), (109), and Fig. 5, anomalous soliton solutions and related their parameters to those of the corresponding stationary states. The single soliton is a special case of a more general 3spin solution, which we have also derived. In the vicinity of a stationary state, both normal and anomalous multi-solitons break up into a sum of single solitons. These single solitons are unstable normal modes in the linear analysis around the stationary state.

The utility of the soliton solutions is that they are explicit and are in terms of elementary functions (exponents), in contrast to the general solution in terms of hyperelliptic functions [29]. At the same time, the dynamics in many physical situations is multi-soliton. The combination of these two factors makes solitons potentially quite useful in various problems in non-stationary superfluidity. Consider, for example, the collisionless dynamics triggered by an abrupt change of the pairing strength. In most cases of interest $\mathbf{L}^{2}(u)$ has only few isolated zeros, while the remaining complex zeros merge into continuous lines[34]. We believe that the latter zeros can be treated as being degenerate and their contribution is therefore multi-soliton. The solution is then a superposition of a (quasi-)periodic few spin solution [29, 30, 33] with a multi-soliton one. Superpositions of this type are referred to as solitons on a (quasi-)periodic background in the soliton theory [53]. In particular, when the system is in the ground state before the coupling change, the collisionless dynamics governed by Eq. (1) can produce asymptotic states with a constant nonzero order parameter or a gapless state $\left[34,35,36\right.$. In these cases $\mathbf{L}^{2}(u)$ has either a single pair of nondegenerate zeros or no such zeros. Therefore, according to the above reasoning, the dynamics leading to these asymptotic states is described by a multi-soliton solution of a normal type for the gapless state and of an anomalous type otherwise.

\section{ACKNOWLEDGEMENTS}

We thank M. Dzero for many stimulating discussions. This research was financially supported by the National Science Foundation award NSF-DMR-0547769 and a David and Lucille Packard Foundation Fellowship for Science and Engineering.

[1] D. Goldhaber-Gordon et al., Nature (London) 391, 156 (1998).

[2] S.M. Cronenwett, T. H. Oosterkamp, and L. P. Kouwenhoven, Science 281, 540 (1998). 
[3] W.G. van der Wiel et al., Science 289, 2105 (2000).

[4] J. Nygård, D. H. Cobden, and P. E. Lindelof, Nature (London) 408, 342 (2000).

[5] A. Kaminski, Yu. V. Nazarov, and L. I. Glazman, Phys. Rev. Lett. 83, 384 (1999).

[6] P. Coleman, C. Hooley, and O. Parcollet, Phys. Rev. Lett. 86, 4088 (2001).

[7] A. Rosch, J. Paaske, J. Kroha, and P.Wölfle, Phys. Rev. Lett. 90, 076804 (2003).

[8] A. Mitra, A. J. Millis, Phys. Rev. B 72, 121102 (2005).

[9] P. Mehta and N. Andrei, Phys. Rev. Lett. 96, 216802 (2006).

[10] T. Schumm et al, Nature Phys. 1, 57 (2005).

[11] M. A. Cazalilla, Phys. Rev. Lett. 97, 156403 (2006).

[12] R. Bistritzer and E. Altman, PNAS 104, 9955 (2007).

[13] V. Gritsev, E. Demler, M. Lukin, A. Polkovnikov, Phys. Rev. Lett. 99, 200404 (2007).

[14] K. Ono and S. Tarucha, Phys. Rev. Lett. 92, 256803 (2004).

[15] A. S. Bracker et al., Phys. Rev. Lett. 94, 047402 (2005).

[16] J. R. Petta et al., Science 309, 2180 (2005).

[17] E. Laird et al., Phys. Rev. Lett. 97, 056801 (2006).

[18] A. V. Khaetskii, D. Loss, and L. Glazman, Phys. Rev. Lett. 88, 186802 (2002).

[19] S. I. Erlingsson and Y. V. Nazarov, Phys. Rev. B 66, 155327 (2002).

[20] I. A. Merkulov, A. L. Efros, and M. Rosen, Phys. Rev. B 65, 205309 (2002).

[21] R. de Sousa and S. Das Sarma, Phys. Rev. B 67, 033301 (2003).

[22] K. A. Al-Hassanieh, V. V. Dobrovitski, E. Dagotto, B. N. Harmon, Phys. Rev. Lett. 97, 037204 (2006).

[23] V. P. Galaiko, Sov. Phys. JETP 34, 203 (1972).

[24] A. F. Volkov and Sh. M. Kogan, Sov. Phys. JETP 38, 1018 (1974).

[25] Yu. M. Gal'perin, V. I. Kozub, and B. Z. Spivak, Sov. Phys. JETP 54, 1126 (1981).

[26] V. S. Shumeiko, Dynamics of electronic system with off-diagonal order parameter and non-linear resonant phenomena in superconductors, Doctoral Thesis, Institute for Low Temperature Physics and Engineering, Kharkov, 1990.

[27] R. A. Barankov, L. S. Levitov, and B. Z. Spivak, Phys. Rev. Lett. 93, 160401 (2004).

[28] M.H.S. Amin, E.V. Bezuglyi, A.S. Kijko, A.N. Omelyanchouk, Low Temp. Phys. 30, 661 (2004).

[29] E. A. Yuzbashyan, B. L. Altshuler, V. B. Kuznetsov, V. Z. Enolskii, J. Phys. A 38, 7831 (2005).

[30] E. A. Yuzbashyan, B. L. Altshuler, V. B. Kuznetsov, V. Z. Enolskii, Phys. Rev. B 72, 220503 (2005).

[31] M. H. Szymanska, B. D. Simons, and K. Burnett, Phys. Rev. Lett. 94 , 170402 (2005).

[32] G. L. Warner and A. J. Leggett, Phys. Rev. B 71, 134514 (2005).

[33] E. A. Yuzbashyan, B. L. Altshuler, and V. B. Kuznetsov, Phys. Rev. B 72, 144524 (2005).

[34] E. A. Yuzbashyan, O. Tsyplyatyev and B. L. Altshuler, Phys. Rev. Lett. 96, 097005 (2006).

[35] R. A. Barankov and L. S. Levitov, Phys. Rev. Lett. 96, 230403 (2006).

[36] E. A. Yuzbashyan and M. Dzero, Phys. Rev. Lett. 96, 230404 (2006).

[37] M. Dzero, E. A. Yuzbashyan, B. L. Altshuler, P. Coleman, Phys. Rev. Lett. 99, 160402 (2007). 
[38] J. Bardeen, L.N. Cooper, and J.R. Schriefer, Phys. Rev. 1081175 (1957).

[39] M.Tinkham, Introduction to Superconductivity, (McGraw-Hill, 1996)

[40] P. W. Anderson, Phys. Rev. 112, 1900 (1958).

[41] E. Abrahams and T. Tsuneto, Phys. Rev. 152, 416 (1966).

[42] V. I. Arnold, Mathematical Methods of Classical Mechanics, Appendix 13, 2nd ed., (Springer-Verlag, New York, 1989).

[43] L. D. Faddeev and L. A. Takhtajan, Hamiltonian methods in the theory of solitons, (Springer-Verlag, Berlin-New York, 1987).

[44] S. P. Novikov, S. V. Manakov, L.P. Pitaevskii, and V. E. Zakharov, Theory of Solitons: The Inverse Scattering Method, (Consultants Bureau, New York, 1984).

[45] R. W. Richardson, J. Math. Phys. 18, 1802 (1977).

[46] L. P. Gorkov, Sov. Phys. JETP 7, 505 (1958).

[47] P. W. Anderson, J. Phys. Chem. Solids 11, 26 (1959).

[48] E. K. Sklyanin, J. Sov. Math. 47, 2473 (1989); Progr. Theoret. Phys. Suppl. 118, 35 (1995).

[49] V. B. Kuznetsov: J. Math. Phys. 33, 3240, (1992).

[50] Indeed, the numerators of $L_{x}(u), L_{y}(u)$, and $L_{z}(u)$ are polynomials in $u$ with real coefficients. Let $c$ be a real (double) zero of $\mathbf{L}^{2}(u)$ and let $a_{x}, a_{y}$, and $a_{z}$ be the remnants from the division of these polynomials by $(u-c)$. Since $c$ is real $a_{x, y, z}$ are also real. It follows from $L_{x}^{2}(u)+L_{y}^{2}(u)+L_{z}^{2}(u)=\mathbf{L}^{2}(u)$ that $a_{x}^{2}+a_{y}^{2}+a_{z}^{2}=0$, i.e. $a_{x}=a_{y}=a_{z}=0$.

[51] Note however that since $\mathbf{L}^{2}(u)$ is conserved by the evolution, the roots of $Q_{2 n}(u)$ are constants of motion.

[52] The frozen separation variables are the solutions of $L_{s}(u)=0$, where $L_{s}(u)$ is given by Eq. (38). Particle-hole symmetry implies $L_{s}(u)=-L_{s}(-u)$ and therefore $\sum_{j=3}^{n-1} u_{j}=0$.

[53] E. A. Kuznetsov and A. V. Mikhailov, Soviet Phys. JETP 40, 855 (1975). 